\title{
1 A photo-switchable assay system for dendrite degeneration and repair in Drosophila 2 melanogaster
}

4 Han-Hsuan Liu ${ }^{1}$, Chien-Hsiang Hsu ${ }^{2}$, Lily, Y. Jan ${ }^{1}$ and Yuh-Nung Jan ${ }^{1}$

1. Howard Hughes Medical Institute, Departments of Physiology, Biochemistry and Biophysics,

7 University of California, San Francisco, San Francisco, CA, United States.

8 2. Department of Pharmaceutical Chemistry, University of California, San Francisco, San

9 Francisco, CA, United States.

\section{ABSTRACT}

12 Neurodegeneration arising from aging, injury or disease has devastating health consequences.

13 Whereas neuronal survival and axon degeneration have been studied extensively, much less is

14 known about how neurodegeneration impacts dendrites. To develop an assay for dendrite

15 degeneration and repair in the Drosophila peripheral nervous system, we used photo-switchable

16 caspase-3 (caspase-LOV) to induce neuronal damage with tunable severity by adjusting

17 illumination duration, thereby revealing cell type-specific responses to caspase-3 induced dendrite

18 degeneration in dendrite arborization (da) neurons. To ask whether mechanisms underlying axon

19 degeneration also govern dendrite degeneration, we tested the involvement of the Wallerian

20 degeneration pathway by examining the effects of expressing the mouse Wallerian degeneration

21 slow $\left(\mathrm{Wld}^{\mathrm{S}}\right)$ protein and knockdown of the Drosophila sterile alpha/Armadillo/Toll-Interleukin

22 receptor homology domain protein (dSarm1) and Axundead (Axed) in class 4 da neurons. Here

23 we report Wld ${ }^{\mathrm{S}}$ expression or knockdown of dSarm1 improved dendrite repair following caspase-

243 induced dendrite degeneration. Whereas both dSarm1 and Axed were required for thermal

25 nocifensive behavior in uninjured animals, Wld ${ }^{\mathrm{S}}$ expression improved the recovery of thermal

26 nocifensive behavior that was impaired by chronic low-level of caspase-LOV activity. By

27 establishing ways to induce graded dendrite degeneration, we uncover a protective role of Wld ${ }^{\mathrm{S}}$ in

28 caspase-3 induced dendrite degeneration and repair. 


\section{INTRODUCTION}

33 Neurodegeneration may cause disabilities that place tremendous burdens on both patients and

34 society at large. While much progress has been made in the study of neuronal survival and axon

35 degeneration, it remains an open question as to how dendrites respond to injuries or

36 neurodegeneration. Dendrite degeneration may result from neurological disorders, traumatic brain

37 injury, aging, and other insults (Kulkarni and Firestein, 2012; Kweon et al., 2017; Mulherkar et

38 al., 2017; Penzes et al., 2011; Xiong et al., 2019). These deleterious changes in dendrite structures

39 impair how neurons receive and process information, likely causing major deficits to neurological

40 function (Mulherkar et al., 2017; Penzes et al., 2011). Elucidating the underlying mechanisms of

41 dendrite degeneration and repair will help to uncover ways to reduce damage and facilitate

42 recovery and thus has important clinical implications. Physiologically relevant and reliable in vivo

43 injury models are key to better understanding how dendrite degeneration may be reduced and to

44 what extent dendrites are capable of repair.

45 Drosophila dendrite arborization (da) neurons are well suited for studying dendrite 46 development, degeneration, and repair. They are sensory neurons in the body wall and the

47 confinement of their dendrites in a primarily two-dimensional space is conducive to live imaging

48 (Jan and Jan, 2010). Based on the dendrite arbor complexity, da neurons are grouped into four

49 classes with class $4 \mathrm{da}(\mathrm{c} 4 \mathrm{da})$ neurons displaying the most complex dendrite arbors (Grueber et al.,

50 2002). Da neurons can sense and initiate response to different harmful sensory modalities. For

51 example, c4da neurons can detect high temperature, harsh mechanical stimulation, noxious

52 chemicals, and harmful short wave-length light (Gorczyca et al., 2014; Hwang et al., 2012; Kim

53 et al., 2012; Xiang et al., 2010; Zhong et al., 2010), whereas class 3 da (c3da) neurons are

54 specialized for sensing gentle mechanical stimulation (Yan et al., 2013). The behavioral readouts

55 of da neurons, such as the fast crawling and rolling escape behaviors initiated by c4da neurons

56 upon high temperature, are well-characterized and can be used for assessments of functional

57 recovery (Babcock et al., 2009; Hwang et al., 2007). Studies that use laser ablation to sever

58 dendrites from the $\mathrm{c} 4 \mathrm{da}, \mathrm{c} 3 \mathrm{da}$ and c1da neuron somata have shown that dendrites can repair

59 themselves. The repair process depends on kinases, electrical activity, extracellular environment,

60 microRNA, and kinetochore proteins (DeVault et al., 2018; Hertzler et al., 2020; Kitatani et al.,

612020 ; Nye et al., 2020; Song et al., 2012; Stone et al., 2014; Thompson-Peer et al., 2016). However,

62 the harsh injury caused by dendrite severing is likely more severe and drastic as compared to insults 
63 induced by neurological disorders, traumatic brain injury, aging, and other insults. Moreover, laser 64 ablation is labor-intensive and hence not suitable for high-throughput screening designed to 65 uncover novel mechanisms. In order to gain insights on how dendrites degenerate and repair, it is 66 desirable to develop an alternative neurodegeneration model that can better simulate how a neuron 67 responds to the insults that it may encounter in its lifetime.

Many conditions can induce neurodegeneration. In this study, we used caspase-3, which acts downstream of various insults, as a switch to initiate neurodegeneration. Activation of caspase-3, an executor for apoptotic cell death, has been observed in neurons exposed to insults such as injury, neurotoxins, and neurodegenerative diseases (Cotman and $\mathrm{Su}$, 1996; Eldadah and Faden, 2000). There are also circumstances where, following caspase-3 activation, neurons stay alive and display degeneration or partial remodeling in dendrites or axons (Erturk et al., 2014;

74 Khatri et al., 2018; Kuo et al., 2006; Simon et al., 2016; Williams et al., 2006). These observations suggest that caspase- 3 could be used as a way to introduce damage on dendrites systematically to elicit neurodegeneration. A recently developed photo-switchable caspase-3, caspase-LOV, provides opportunities to test whether a controllable caspase- 3 could be a versatile tool to induce neurodegeneration with diverse outcomes ranging from apoptosis to repair (Smart et al., 2017). In this system, a light-oxygen-voltage-sensing domain (LOV domain) is inserted into the intersubunit

80 linker of human caspase-3 (Smart et al., 2017). Illumination with $450 \mathrm{~nm}$ light activates this photo-

81 switchable caspase-3, and the activation only lasts for the duration of illumination. This reversible

82 feature of caspase-LOV makes it possible to adjust the degree of caspase-3 activity during a 83 specific time window (Smart et al., 2017).

84 Wallerian degeneration is an evolutionarily conserved process to clear distal axons after 85 axon injury. This process can be delayed by neuronal expression of the mouse Wallerian 86 degeneration slow $\left(\mathrm{Wld}^{\mathrm{S}}\right.$ ) protein in both mice and flies (Hoopfer et al., 2006; Lunn et al., 1989;

87 MacDonald et al., 2006). Wld ${ }^{\mathrm{S}}$ can also partially protect axon degeneration following trophic 88 deprivation and dendrite pruning during metamorphosis, both of which are caspase-3 dependent 89 (Schoenmann et al., 2010; Tao and Rolls, 2011). Caspase-3 independent dendrite degeneration 90 induced by injury or phosphatidylserine (PS) exposure could be delayed with Wld ${ }^{\mathrm{S}}$ as well (Ji et 91 al., 2021; Sapar et al., 2018). Interestingly, a study using both mouse and Drosophila models raises 92 the possibility that $\mathrm{Wld}^{\mathrm{S}}$ and caspase act in parallel during dendrite pruning, because Wld ${ }^{\mathrm{S}}$ does 93 not supress caspase activity (Schoenmann et al., 2010). Loss-of-function mutations in Drosophila 
94 Toll receptor adaptor proteins, the sterile alpha/Armadillo/Toll-Interleukin receptor homology 95 domain protein (dSarm1) and Axundead (Axed), both of which are involved in the Wallerian 96 degeneration pathway, afford protection for axon degeneration induced by injury but not axon

97 degeneration during developmental pruning or apoptotic cell death (Neukomm et al., 2017; 98 Osterloh et al., 2012). The suppression of injury-induced axon degeneration can be achieved by 99 knocking down expression of dSarm1 with RNAi as well (Gerdts et al., 2013). Deletion of dSarm1

100 protects injury- and PS-induced dendrite degeneration (Ji et al., 2021), whereas deletion of Axed 101 only partially protects the injury-induced dendrite degeneration but does not affect PS-induced 102 dendrite degeneration ( $\mathrm{Ji}$ et al., 2021). It is unclear whether these proteins involved in the Wallerian 103 degeneration pathway play any roles in caspase-3 dependent dendrite degeneration and repair.

104 To elucidate the cellular mechanism of dendrite degeneration and repair, we used the 105 photo-switchable caspase-3 to induce varying degrees of dendrite degeneration in Drosophila 106 larval da neurons and monitored the repair process afterward. We found that the caspase-3 107 dependent dendrite degeneration in da neurons was worsened by prolonging the illumination, and 108 dendrite repair was evident with attenuated activation of caspase-3. We observed cell type-specific 109 responses to caspase-3 induced dendrite degeneration in da neurons. Expression of mouse Wld ${ }^{\mathrm{S}}$ in 110 c4da neurons resulted in longer and more numerous dendrites during caspase-3 induced dendrite 111 degeneration and during development as well. Similarly, knockdown of dSarm1 or Axed, two 112 factors involved in Wallerian degeneration, increased survival of neurons following caspase-LOV 113 activation. Additionally, knockdown of dSarm1 led to longer dendrites both during development 114 and following caspase-LOV activation. Reduced expression of Axed did not affect the dendrite 115 structure during development or following caspase-LOV activation. We further showed that the 116 compromised thermal nocifensive behavior caused by chronic low-level of caspase-LOV activity 117 in c4da neurons can be partially rescued with $\mathrm{Wld}^{\mathrm{S}}$ expression but not with knockdown of dSarm 1 118 or Axed.

\section{RESULTS}

121 Caspase-LOV activation of different durations initiates graded dendrite degeneration in 122 sensory neurons

123 Among larval da neurons, c4da neurons display the most complex dendrite structures (Grueber et 124 al., 2002). Their dendrites actively grow in length, scale in size to extend coverage area, and 
125 continue adding new tips throughout larval development (Grueber et al., 2002; Parrish et al., 2009;

126 Williams and Truman, 2005). In this study, we sought to determine to what extent c4da neurons

127 can recover from caspase-3 induced degeneration following transient activation of a photo-

128 switchable caspase-3, caspase-LOV. The amount of illumination is known to correlate with the

129 amount of caspase-3 activity which can effectively induce dendrite degeneration followed by

130 apoptosis in several type of cells including c4da neurons (Smart et al., 2017).

131 Given that the activation of caspase-LOV can be easily controlled by adjusting the intensity

132 and the duration of illumination, we began our study by monitoring dendrite degeneration

133 following caspase-LOV activation for 2 hours (h), 30 minutes (min), or 10 min. We used a

134 membrane tethered tdTomato (UAS-CD4-tdTOM) driven by the ppk-GAL4 to label the plasma

135 membrane of c4da neurons for visualization of individual dendrite arbors. Freely moving larvae

136 were illuminated for various durations at $48 \mathrm{~h}$ after egg laying (AEL) on transparent agar plates

137 and then transferred back to a dark environment. We performed time-lapse imaging to monitor the

138 dendrite structure of the same c4da neuron, ddaC, $24 \mathrm{~h}$ and $72 \mathrm{~h}$ following illumination with blue

139 LED (Figure 1A). The $24 \mathrm{~h}$ and $72 \mathrm{~h}$ imaging timepoints provide snap shots for the early and late

140 stages of caspase-3 induced dendrite degeneration and subsequent repair, as indications for the

141 acute and continuing response to the degeneration, respectively.

To facilitate the quantification of complex morphology of c4da neurons in this study, we

143 built a deep learning model based on the U-Net architecture (Ronneberger et al., 2015) which has

144 been widely used for biomedical image segmentation, including detecting dendrite branch

145 terminals of da neurons (Kanaoka et al., 2019). We applied our model to automatically segment

146 dendrite structure from microscopy images and retrieve segmentation masks containing the full

147 reconstruction of the dendrite arbors of neurons. Segmentation masks of individual neurons were

148 then used to measure different parameters of neuronal morphology, including total dendrite length,

149 total dendrite tip numbers, percentage of territory covered, and dendrite complexities assessed with

150 Sholl analysis. We validated that the dendrite structures segmented by our model and found that

151 they were comparable to manual reconstruction and achieved high Dice coefficient, a commonly

152 used spatial overlap index for evaluating segmentation quality (Zou et al., 2004) (Figure 1 - figure

153 supplement $1 A$ ). To further evaluate the model performance, we compared parameters of neuronal

154 morphology measured from model-predicted segmentation with those derived from manual

155 reconstruction by using the images of c4da neurons acquired in Figure 1. With post-processing to 
156 fill in gaps and remove small fragments (see Methods), we observed high correlation for both tip numbers $\left(\mathrm{R}^{2}=0.97\right)$ and total dendrite length $\left(\mathrm{R}^{2}=0.99 ;\right.$ Figure 1 -figure supplement $\left.1 B, C\right)$. $1 B, C)$. Shortening the caspase-LOV activation to $30 \mathrm{~min}$ allowed the average survival rate for 160 illuminated neurons to reach $80 \%$. With 10 min caspase-LOV activation, almost all neurons 161 survived for at least $72 \mathrm{~h}$ (Figure 1C). Using the deep learning-based model, we quantified the 162 dendrite structures of c4da neurons that were either kept in the dark or illuminated for a duration 163 ranging from $10 \mathrm{~min}$ to $2 \mathrm{~h}$. Activation of caspase-LOV for $2 \mathrm{~h}$ caused the reduction of total 164 dendrite length, tip numbers, dendrite complexity, and percentage of territory covered, both at 24 $165 \mathrm{~h}$ and at $72 \mathrm{~h}$ following caspase-LOV activation (Figure $1 \boldsymbol{B}, \boldsymbol{D}, \boldsymbol{E}, \boldsymbol{F}, \boldsymbol{G}, \boldsymbol{H}$ ). The total dendrite length, 166 tip numbers, and dendrite complexity decreased progressively with increasing durations of 167 illumination, while the percentage of territory covered was affected at $72 \mathrm{~h}$ after 30 min 168 illumination (Figure $\mathbf{B}, \boldsymbol{D}, \boldsymbol{E}, \boldsymbol{F}, \boldsymbol{G}, \boldsymbol{H}$ ). Neurons exposed to $30 \mathrm{~min}$ of blue LED illumination 169 displayed significantly shorter and fewer dendrites compared to those exposed to $10 \mathrm{~min}$ 170 illumination. The basal activity of caspase-LOV in the dark (dark) led to reduced dendrite arbor 171 length, tip numbers, and dendrite complexity at the $24 \mathrm{~h}$ and $72 \mathrm{~h}$ timepoints compared to the 172 animals without caspase-LOV expression (control) (Figure $1 \boldsymbol{B}, \boldsymbol{D}, \boldsymbol{E}, \boldsymbol{G}, \boldsymbol{H}$ ). The percentage of territory covered by dendrites is not affected by caspase-LOV expression if the animals were kept

174 in the dark (Figure $\mathbf{1 F}$ ). With $30 \mathrm{~min}$ and $2 \mathrm{~h}$ of caspase-LOV activation, there were overall 175 reductions in both dendrite length and tip numbers (Figure $\mathbf{2 A , B}$ ). Interestingly, there were still 176 increases in the dendrite length $24-72 \mathrm{~h}$ after the $10 \mathrm{~min}$ illumination (Figure $2 A$ ), even though 177 the total dendrite tip numbers were reduced (Figure 2B), suggesting that c4da neurons can 178 continue to grow after experiencing caspase-LOV activation. These changes could be a 179 combination of normal dendrite growth, dendrite degeneration, and repair.

180 To further examine the dendrite elimination and addition of c4da neurons, we analyzed the 181 dendrite dynamics in the tip numbers over a period of $48 \mathrm{~h}$ following caspase-LOV activation. We 182 compared the dendrite structure between the $24 \mathrm{~h}$ and $72 \mathrm{~h}$ timepoints and used the dendrite arbor 183 at $24 \mathrm{~h}$ following illumination as the backbone to generate a "transition state arbor" which 184 contained only dendrites observed at both $24 \mathrm{~h}$ and $72 \mathrm{~h}$. Then, we subtracted the number of tips 185 of the "transition state arbor" from that at $24 \mathrm{~h}$ to give a measure of the eliminated dendrites (those 186 dendrite branches only observed at $24 \mathrm{~h}$ ), and from that at $72 \mathrm{~h}$ to give a measure of the newly 
187 added branches (those dendrite branches only observed at $72 \mathrm{~h}$ ) (Figure 2 C). The percentage of

188 eliminated dendrite tips was calculated by dividing the number of eliminated dendrites by the total

189 number of dendrite tips measured at $24 \mathrm{~h}$. The percentage of added dendrite tips was calculated by

190 dividing the number of newly added dendrites by the total number of dendrite tips measured at 72

$191 \mathrm{~h}$.

192 We found that dendrite elimination and addition took place concurrently in individual

193 neurons following caspase-LOV activation (Figure 2D,E). As the duration of Caspase-3

194 activity increased, the percentage of eliminated dendrite tips increased and the percentage of added

195 dendrite tips decreased. Interestingly, even though caspase-LOV activation for 30 min caused

196 reduction in total dendrite length and tip numbers, there were still new branches added following

197 dendrite degeneration. The reduction in total tip numbers following 10 min or 30 min illumination

198 (Figure $2 B$ ) resulted from the significantly greater increase in elimination (Figure 2 D) than

199 addition of dendrite branches (Figure 2 E). C4da neurons expressing caspase-LOV but kept in the

200 dark were comparable with c4da neurons not expressing caspase-LOV based on the percentage of

201 eliminated dendrite tips (Figure 2D) though the former had a higher percentage of newly added

202 dendrites (Figure 2E).

203 Taken together, we found that neurons can survive 10-30 min of caspase-LOV activation

204 through illumination, and their dendrites continue to grow in length with addition of new tips to

205 the remaining dendrite arbors. Most of the neurons failed to survive following caspase-LOV

206 activation for longer than $2 \mathrm{~h}$ and showed severe dendrite degeneration before dying. By making

207 use of the varying levels of degeneration induced by different durations of illumination, we can

208 search for machineries used for neuroprotection to improve dendrite degeneration, repair or

209 neuronal survival following caspase-3 induced degeneration.

211 Class I ddaE neurons can withstand transient caspase-LOV activation and repair dendrite 212 damage

213 Class I da (c1da) neurons and class 3 da (c3da) neurons differ from c4da neurons in dendritic 214 morphology, growth dynamics and physiological function. To ask whether their response to 215 caspase-3 induced regeneration and repair is also different from that of c4da neurons, we first 216 examined c1da neurons, which have the simplest dendrite arbor among all classes of da neurons.

217 C1da neurons establish their dendrite arbor early in development and only extend existing branches 
218 in length without adding new branches in late larval development (Grueber et al., 2002; Williams 219 and Truman, 2005). They are able to initiate regeneration after dendrotomy as are c4da neurons 220 (Sugimura et al., 2003; Tao and Rolls, 2011; Thompson-Peer et al., 2016).

To assess how c1da neurons would react to caspase-3 induced degeneration, we labeled 222 the c1da ddaE neuron with UAS-CD4-tdTOM driven by the GAL4-21 (Grueber et al., 2003a) and 223 used the same paradigm described in Figure 1A. C1da neurons can survived 30 min activation of 224 caspase-LOV, whereas about 10\% of clda neurons imaged were found dead $72 \mathrm{~h}$ following 225 caspase-LOV activation for $2 \mathrm{~h}$ (Figure 3A,B). Caspase-LOV activity in the dark (dark) 226 significantly reduced dendrite length at $72 \mathrm{~h}$ after illumination (Figure $3 \mathrm{C}$ ). The dendrite tip 227 numbers of clda neurons expressing caspase-LOV and maintained in the dark (dark) were fewer 228 than those of c1da neurons without caspase-LOV expression (control) (Figure 3D). Both $30 \mathrm{~min}$ 229 and $2 \mathrm{~h}$ caspase-LOV activation impaired dendrite structures (Figure 3A,C,D). Caspase-LOV 230 activation for $2 \mathrm{~h}$ induced more drastic reductions in both total dendrite length and tip numbers at $23172 \mathrm{~h}$ after illumination, as compared to $30 \mathrm{~min}$ of caspase-LOV activation (Figure $3 \boldsymbol{A}, \boldsymbol{C}, \boldsymbol{D}$ ). The 232 increase in dendrite length (Figure $3 \boldsymbol{E}$ ) and total tip numbers (Figure $3 \boldsymbol{F}$ ) over the $24-72 \mathrm{~h}$ period 233 following $2 \mathrm{~h}$ of caspase-LOV activation was significantly less than dark and 30 min of caspase234 LOV activation.

235 We next looked into the dendrite dynamics and quantified dendrite tip elimination and 236 addition as we did for c4da neurons. Similar to previous reports on the limited increase in dendrite 237 tips after early development (Stone et al., 2014; Sugimura et al., 2003), we found that the c1da 238 ddaE neurons without caspase-LOV (control) had 7\% and 4\% tips added and eliminated, 239 respectively (Figure $3 \boldsymbol{F}, \boldsymbol{G}$ ). Caspase-LOV activity in the dark did not significantly alter the 240 percentage of addition or elimination of dendrite tips. Caspase-LOV activation for $30 \mathrm{~min}$ or $2 \mathrm{~h}$ 241 increased the percentage of eliminated dendrite tips (Figure 3G). There was a robust increase inthe 242 percentage of added dendrite tips of clda neurons expressing caspase-LOV following $30 \mathrm{~min}$ 243 illumination compared to those kept in the dark (Figure 3H). This robust increase in the percentage 244 of added dendrite tips was not observed in c1da neurons following $2 \mathrm{~h}$ illumination nor in c4dan 245 neurons following any durations of illumination tested in this study (Figure 2 E and Figure $3 \boldsymbol{H}$ ).

246 Thus, there appears to be a regrowth program unique for c1da neurons that is initiated following 24730 min caspase-LOV activation - a program that is not evident following severe degeneration 248 induced by $2 \mathrm{~h}$ caspase-LOV activation. 
250 Class III ddaE neurons can withstand transient caspase-LOV activation induced dendrite damage

C3da neurons have signature bushy tertiary branches enriched in actin (Nagel et al., 2012;

253 Tsubouchi et al., 2012). In contrast to the c4da ddaC and c1da ddaE neurons, c3da neurons do not

254 persist after metamorphosis (Shimono et al., 2009; Williams and Truman, 2005). To test whether

255 they can survive caspase-3 activation, we expressed caspase-LOV in the c3da ddaF neurons and

256 imaged these neurons at 24 and $72 \mathrm{~h}$ after illumination. We labeled the c3da ddaF neurons with

257 UAS-CD4-tdTOM driven by the GAL4 ${ }^{19-21}$ along with Repo-Gal80 to eliminate the expression in

258 glial cells (Awasaki et al., 2008; Xiang et al., 2010). The c3da ddaF neurons can survive 30 min

259 but not $2 \mathrm{~h}$ caspase-LOV activation (Figure $4 \boldsymbol{A}, \boldsymbol{B}$ ). The survival rate of c3da ddaF neurons was

260 significantly reduced to $71 \%$ following 2 h caspase-LOV activation (Figure $4 \boldsymbol{A}, \boldsymbol{B})$. Caspase-LOV

261 activity in the dark (dark) induced significant reduction in dendrite tip numbers but did not alter

262 the total dendrite length compared to c3da neurons without caspase-LOV (control) (Figure 4C,D).

263 Both $30 \mathrm{~min}$ and $2 \mathrm{~h}$ of caspase-LOV activation in c3da ddaF neurons led to reduction in total

264 dendrite length and tip numbers (Figure 4C,D). Caspase-LOV activity in the dark significantly

265 reduced the increase in dendrite length (Figure $4 \boldsymbol{E}$ ) but did not significantly alter the dendrite tip

266 numbers (Figure $\mathbf{4 F}$ ). The dendrite length and tip numbers still exhibited increases over the 24-

$26772 \mathrm{~h}$ period following $30 \mathrm{~min}$ or $2 \mathrm{~h}$ of caspase-LOV activation (Figure $\mathbf{4 E}, \boldsymbol{F}$ ). Moreover, there

268 were significant increases in the percentage of eliminated tips and significant decreases in the

269 percentage of added new tips following $2 \mathrm{~h}$ of caspase-LOV activation (Figure $4 \boldsymbol{G}, \boldsymbol{H}$ ). Thus, c3da

270 ddaF neurons also appear to have a class-specific response to caspase-3 induced dendrite

271 degeneration. They do not initiate regrowth following mild degeneration as observed in c1da ddaE

272 neurons. Instead, there was greater degeneration of c3da neuronal dendrites following longer

273 caspase activation, similar to what we observed in c4da neurons. The c3da ddaF neurons differ

274 from c4da ddaC neurons in that they do not show any increase in the percentage of added dendrite

275 tips with caspase-LOV activity in the dark (Figure $2 \boldsymbol{E}$ and Figure $4 \boldsymbol{H}$ ) and they continue to grow

276 in length and add new tips following caspase-LOV activation (Figure $2 A, B$ and Figure $4 \boldsymbol{E}, \boldsymbol{F}$ ).

278 Wld $^{\mathrm{S}}$ protects c4da neurons from caspase-3 dependent dendrite degeneration 
This new degeneration assay system can be used to address questions such as how caspase-LOV

280 activation induces dendrite degeneration, how neurons manage to survive from transient caspase-

281 LOV activation, and how neurons repair their damaged dendrites. We decided to focus on c4da

282 neurons because they have the most complex dendrites among the da neurons (Grueber et al., 2002)

283 and there are established behavioral assays to assess their sensory functions (Babcock et al., 2009;

284 Hwang et al., 2007).

285 We generated caspase-tester flies expressing the ppk-tdGFP transgene to monitor the 286 dendrite morphology of c4da neurons with caspase-LOV expressed via ppk-GAL4. These tester 287 flies were crossed with either RNAi flies or flies harboring other transgenes of interest. To select 288 illumination conditions, we first examined the degree of dendrite degeneration and repair in c4da 289 neurons labeled with ppk-tdGFP and expressing caspase-LOV and luciferase (control) via ppk290 GAL4. We found that $91 \%$ of the neurons survived the $10 \mathrm{~min}$ illumination, and the survival rate 291 dropped to $22 \%$ following $30 \mathrm{~min}$ illumination (Figure 5 - figure supplement 1A,B). We 292 suspected that the lower survival rate following $30 \mathrm{~min}$ illumination here compared to Fig. 1 is due 293 to the stronger ppk-Gal4 used for caspase-tester flies, which has an insertion site different from 294 the ppk-Gal4 used in Fig. 1. The 10 min caspase-LOV activation decreased dendrite length and 295 dendrite tip numbers at $24 \mathrm{~h}$ and $72 \mathrm{~h}$ after illumination and degeneration was worse when 296 activation of caspase-LOV extended to $30 \mathrm{~min}$ (Figure 5 - figure supplement 1 A,C,D,E). The 297 percentage of territory covered was not affected in the neurons that survived the $10 \mathrm{~min}$ or $30 \mathrm{~min}$ 298 illumination (Figure 5 - figure supplement 1A,E).

Wld ${ }^{S}$ has been found to be beneficial for protection against injury-induced dendrite degeneration, PS-induced dendrite degeneration, and developmental dendrite pruning (Ji et al., 2021; Sapar et al., 2018; Schoenmann et al., 2010; Tao and Rolls, 2011). It is unclear whether

$302 \mathrm{Wld}^{\mathrm{S}}$ is also involved in early dendrite development or caspase-3 dependent dendrite degeneration 303 and repair. During early dendrite development, neurons expressing $\mathrm{Wld}^{\mathrm{S}}$ displayed mild but 304 significant increases in total dendrite length and tip numbers with no changes in the percentage of 305 territory covered (Figure $\mathbf{5} \boldsymbol{A}, \boldsymbol{B}, \boldsymbol{C}, \boldsymbol{D})$ ). Using the caspase-tester flies, we examined the functions of $306 \mathrm{Wld}^{\mathrm{S}}$ expression in caspase-3 dependent dendrite degeneration and repair. To maintain comparable 307 expression levels of UAS-caspase-LOV driven by ppk-GAL4 in neurons with or without Wld ${ }^{\mathrm{S}}$ 308 expression, we include UAS-mIFP-2A-HO1 transgene in the control group. The transgenic flies 309 harboring UAS-mIFP-2A-HO1, which had a wildtype genetic background similar to that of flies 
310 with UAS-Wld ${ }^{\mathrm{S}}$, expressed monomeric infrared fluorescent proteins (IFP) and Heme Oxygenase

3111 Proteins (HO1) driven by Gal4. With caspase-3 induced neurodegeneration, neurons expressing

$312 \mathrm{Wld}^{\mathrm{S}}$ were comparable to control at $24 \mathrm{~h}$ following illumination, but these neurons retained

313 significantly longer dendrites and more numerous dendrite tips at $72 \mathrm{~h}$ following $10 \mathrm{~min}$ of

314 caspase-LOV activation (Figure $5 \boldsymbol{E}, \boldsymbol{F}, \boldsymbol{G})$. The protection in dendrite structure afforded by Wld ${ }^{\mathrm{S}}$

315 was already evident at $24 \mathrm{~h}$ following $30 \mathrm{~min}$ of caspase-LOV activation, as revealed by the longer

316 dendrites and more numerous dendrite tips (Figure $\mathbf{5 I , K , L}$ ). Wld ${ }^{\mathrm{S}}$ expression did not alter the

317 percentage of territory covered following $10 \mathrm{~min}$ or $30 \mathrm{~min}$ caspase-LOV activation (Figure

$318 \boldsymbol{5 H}, \boldsymbol{M})$. With 30 min illumination, neuronal survival was enhanced by $\mathrm{Wld}^{\mathrm{S}}$ expression in c4da

319 neurons (Figure 5J). These results suggest that expression of $\mathrm{Wld}^{\mathrm{S}}$ can protect $\mathrm{c} 4 \mathrm{da}$ neurons from

320 caspase-3 induced dendrite degeneration.

Knockdown of Axed and dSarm1 are neuroprotective with dSarm1 playing a role in dendrite degeneration and repair

324 Besides Wld ${ }^{\mathrm{S}}$, dSarm1 and Axed are two additional players involved in the Wallerian degeneration

325 pathway. It is unknown how dSarm1 and Axed are involved in early dendrite development and 326 caspase-3 dependent dendrite degeneration and repair in c4da neurons. Hence, we use ppk-GAL4 327 to drive the expression of luciferase (control) or RNAi targeting dSarm1 or Axed in c4da neurons.

328 During early dendrite development, knocking down dSarm1 in c4da neurons resulted in longer


330 Axed had no significant effect in early dendrite development (Figure $6 A, B, C, D)$. During caspase-

3313 induced dendrite degeneration, neurons with reduced dSarm1 expression had longer and more 332 numerous dendrites and a higher percentage of territory covered with dendrite at $72 \mathrm{~h}$ after $10 \mathrm{~min}$ 333 illumination (Figure $\mathbf{6 E}, \boldsymbol{F}, \boldsymbol{G}, \boldsymbol{H}$ ). Similar effects on dendrite structure were observed at $24 \mathrm{~h}$ 334 following $30 \mathrm{~min}$ illumination (Figure 6I,K,L,M). Knockdown of Axed did not affect dendrite


336 with reduced dSarm1 or Axed expression had a higher survival rate (Figure 6J). These results 337 indicate that knockdown of dSarm1 or Axed in c4da neurons can increase neuronal survival 338 following caspase-3 induced degeneration, whereas knockdown of dSarm1 but not Axed can 339 protect dendrite structure from caspase-3 induced degeneration. Moreover, dSarm1 is also 340 involved in early dendrite development for regulation of dendrite elongation. 
Wld ${ }^{\mathrm{S}}$ can partially rescue caspase 3 -induced neurodegeneration and impairment of thermal nocifensive behavior

344 The chronic low-level caspase-LOV activity in the dark caused mild but significant dendrite 345 degeneration during early larval development (Figure $\mathbf{1 A}, \boldsymbol{B}, \boldsymbol{D}, \boldsymbol{E}, \boldsymbol{F}, \boldsymbol{G}, \boldsymbol{H}$ ). This mild degeneration continued throughout development up to the stage of wandering larvae (Figure 7A). These c4da neurons displayed impaired dendrite structure including shorter dendrites, fewer dendrite tips, and

348 a lower percentage of territory covered (Figure $7 \boldsymbol{A}, \boldsymbol{B}$ ). We wondered whether these neurons with 349 dendrite degeneration can fulfill normal sensory function. As nociceptive neurons, c4da neurons 350 are required for the aversive rolling behavior when larvae encounter nocifensive stimuli such as 351 high temperature (Babcock et al., 2009; Hwang et al., 2007). To test whether caspase-3 induced neurodegeneration affects the neuronal function, we examined the thermal nocifensive behavior

353 in wandering larvae kept in the dark with or without caspase-LOV expression at two nocifensive 354 temperatures, $46^{\circ} \mathrm{C}$ for tests of insensitivity, and $42^{\circ} \mathrm{C}$ for testing hypersensitivity (Honjo et al., 355 2016). We measured the time it took for an individual larva to initiate the rolling behavior within 35620 seconds (s) of contacting the thermal probe at high temperature. We also quantified the 357 percentage of non-responders (larvae that did not respond within $20 \mathrm{~s}$ ). We found that larvae kept 358 in the dark with chronic low-level caspase-LOV activity in c4da neurons took longer to initiate 359 rolling behavior to escape the high temperature and a higher percentage of them were non360 responders (Figure 7 C,D).

Having found that $\mathrm{Wld}^{\mathrm{S}}$ expression in c4da neurons afforded protection from caspase-3

362 induced dendrite degeneration, we tested for its effect on the caspase-3 induced deficiency in the 363 thermal nocifensive behavior. Without caspase-LOV, Wld ${ }^{\mathrm{S}}$ expression in c4da neurons slightly 364 increased the number of dendrite tips of c4da neurons in the wandering larvae (Figure $8 \boldsymbol{A}, \boldsymbol{B})$ but 365 did not change their response time or the percentage of non-responders in the thermal nocifensive 366 behavior (Figure 8C,D). We then examined the thermal nocifensive behavior of wandering larvae 367 expressing caspase-LOV along with UAS-mIFP-2A-HO1 (control) or UAS-Wld ${ }^{\mathrm{S}}\left(\mathrm{Wld}^{\mathrm{S}}\right.$ ). With 368 chronic low-level caspase-LOV activity in the dark, Wld ${ }^{\mathrm{S}}$ expression resulted in longer and more 369 numerous dendrite tips but with a smaller percentage of territory covered by dendrites (Figure $370 \boldsymbol{8} \boldsymbol{E}, \boldsymbol{F})$. Moreover, $\mathrm{Wld}^{\mathrm{S}}$ partially rescue the caspase-3 induced impairment in thermal nocifensive 371 behavior. Wld ${ }^{\mathrm{s}}$ expression in c4da neurons reduced the averaged time to respond to a $46^{\circ} \mathrm{C}$ heat 
372 probe (Figure 8G). It also reduced the percentage of non-responders in larvae expressing caspase-

373 LOV and kept in the dark (Figure $8 \boldsymbol{H}$ ). These findings indicate that $\mathrm{Wld}^{\mathrm{S}}$ expression in c4da

374 neurons not only afforded preservation in dendrite structures but also protected neuronal functions

375 critical for behavioral response to nociceptive stimuli.

376 While knockdown of dSarm1 or Axed did not affect dendrite structure of c4da neurons in

377 the wandering larvae (Figure 9A,B), dSarm1 knockdown delayed the behavioral response to

378 contacts with a probe heated to $46^{\circ} \mathrm{C}$ (Figure 9C). Knockdown of either dSarm1 or Axed increased

379 the population of non-responders upon encounter with a probe at the nocifensive temperature of

$38042^{\circ} \mathrm{C}$ (Figure 9D). With mild degeneration induced by the chronic low-level caspase-LOV activity

381 in the dark throughout larval development, dSarm1 knockdown caused a small increase in the

382 percentage of territory covered by c4da neuron dendrites in the wandering larvae (Figure 9 E,F),

383 while RNAi knockdown of Axed did not affect the degeneration of dendrite structure (Figure

$384 \boldsymbol{9 E}, \boldsymbol{F})$. Notably, knockdown of dSarm1 or Axed reduced the thermal nocifensive behavior of



386 in c4da neurons took longer to avoid the probe heated to $42^{\circ} \mathrm{C}$ (Figure $9 G$ ). Knockdown of dSarm 1

387 or Axed in c4da neurons increased the percentage of non-responders when stimulated with a probe

388 heated to $42^{\circ} \mathrm{C}$ or $46^{\circ} \mathrm{C}$ (Figure $9 H$ ). These results indicate that knockdown of dSarm1 or Axed in

389 c4da neurons impaired the thermal nocifensive behavior of larvae during development and further

390 exasperated the deficient thermal nocifensive behavior owing to caspase-3 induced degeneration

391 of c4da neurons.

\section{DISCUSSION}

394 In this study, we established a new neurodegeneration and repair assay system with the photo-

395 switchable caspase-3, caspase-LOV, to elucidate the mechanisms underlying dendrite 396 degeneration and repair. To characterize the caspase-3 induced neurodegeneration, we focused on 397 the dendrite morphology for different classes of da neurons and observed cell type-specific cellular 398 responses. We also examined the c4da neurons-mediated thermal nocifensive behavior to reveal 399 the functional consequence of neurodegeneration. We found that $\mathrm{Wld}^{\mathrm{S}}$, a key molecule involved 400 in the Wallerian axon degeneration, can protect dendrite structure and reduce the impairment of 401 thermal nocifensive behavior caused by caspase-LOV activation in c4da neurons. Knockdown of 402 dSarm1 reduced the caspase-3 induced loss in dendrite structure, whereas knockdown of Axed did 
403 not affect dendrite degeneration. Knockdown of dSarm1 or Axed led to impaired thermal

404 nocifensive behavior with or without caspase-3 induced degeneration of c4da neurons. Along with

405 the previously established laser severing injury model, our new model with adjustable caspase-

406 LOV activation provides a useful platform to identify regulators and to improve our understanding

407 of dendrite degeneration and repair.

Cell type-specific cellular responses upon caspase-3 induced dendrite degeneration

410 We examined how three different classes of da neurons react to caspase-LOV activation and found

411 cell type-specific responses to caspase-3 induced dendrite degeneration. Dendrites of c1da, c3da

412 and c4da neurons all exhibit more severe degeneration following longer duration of caspase-LOV

413 activation. The survival rates of these three classes of da neurons also decreased with longer

414 caspase-LOV activation. However, these da neurons differ in the dynamic dendrite changes over

415 the 24-72 $\mathrm{h}$ period following illumination. Remarkably, c1da neurons displayed an increased

416 percentage of added dendrite tips number following $30 \mathrm{~min}$ but not $2 \mathrm{~h}$ of caspase-LOV activation

417 compared to neurons kept in the dark. This reactivation of the growth program is exhibited by c1da

418 neurons but not $\mathrm{c} 3 \mathrm{da}$ or $\mathrm{c} 4 \mathrm{da}$ neurons. The $\mathrm{c} 3 \mathrm{da}$ neurons continued to grow in dendrite length and

419 tip numbers over the $24-72 \mathrm{~h}$ period following $30 \mathrm{~min}$ and $2 \mathrm{~h}$ of caspase-LOV activation, as

420 control c3da neurons did. In contrast, caspase-LOV activation for $2 \mathrm{~h}$ induced significant changes

421 in dendrite length and tip numbers in both c1da and c4da neurons. Unique to c4da neurons is an

422 increase in the percentage of added dendrite tips with caspase-LOV activity in the dark.

Extensive studies in da neurons revealed the cell-type specific dendrite morphology, gene

424 expressions, dendrite remodeling and injury responses (Grueber et al., 2002; Jan and Jan, 2010;

425 Shimono et al., 2009; Song et al., 2012; Thompson-Peer et al., 2016). Our data further suggest that

426 different classes of da neurons are also equipped with specialized mechanism to handle caspase-3

427 induced neurodegeneration. By including different classes of da neurons in our study, we aim for

428 a more comprehensive survey of how to protect dendrites from degeneration and improve recovery

429 of neuronal functions. For example, future studies of c1da neurons could elucidate the growth

430 programs reactivated following degeneration and assess whether such programs can be transferred

431 to other cell types. As to c3da neurons, it would be of interest to investigate how they can withstand

432 the caspase-LOV activation without halting their growth. 


\section{Protection afforded by Wld ${ }^{\mathrm{S}}$ may vary with the degree of neurodegeneration}

435 The Wallerian degeneration pathway important for axon degeneration serves as a prominent target

436 for therapy. In this study, we focused on the impacts of neurodegeneration on dendrites, which

437 together with axons are responsible for maintaining neuronal functions. Our study of the impact

438 of caspase-3 induced neurodegeneration on dendrite morphology and thermal nocifensive behavior

439 reveals intriguing involvement of the Wallerian degeneration pathway. We found that with 10-30

440 min caspase-LOV activation, $\mathrm{Wld}^{\mathrm{S}}$ can partially rescue the caspase-3 induced deficiency in

441 dendrite structure and neuronal survival. Wld ${ }^{\mathrm{S}}$ also afforded protection for the impaired dendrite

442 structure and thermal nocifensive behavior caused by the chronic low-level caspase-LOV activity

443 in the dark. Interestingly, with continuous activation of the photo-switchable caspase-3 via

444 illumination for days, a much stronger perturbation employed in a previous study, Wld ${ }^{\mathrm{S}}$ fails to

445 rescue the survival of flies with neuronal expression of caspase-LOV (Smart et al., 2017). This

446 suggests that the ability of $\mathrm{Wld}^{\mathrm{S}}$ to provide protection may depend on the level of caspase-LOV

447 activation in a neuron. Whether different mechanisms are used for dendrite degeneration or repair

448 in neurons experiencing different levels of caspase-LOV activation is an interesting question that

449 can be explored using this tunable neurodegeneration model in the future.

\section{Multiple roles of $\mathrm{Wld}^{\mathrm{S}}$ in caspase-3 induced degeneration and repair}

452 The preservation of neuronal function by $\mathrm{Wld}^{\mathrm{S}}$ following caspase-LOV activation may result from 453 the retained dendrite structures and/or axons, or a complex combination of different factors. In this 454 study, we did not examine the caspase-3 induced axon degeneration and repair. The axons of the 455 da neurons project deep into the ventral nerve cord and connect with central neurons to form 456 circuits required for the avoidance behavior. These axons form bundles, while the dendrite arbors 457 of da neurons display readily discernible patterns. To study caspase-3 induced axon degeneration 458 and repair, future studies could examine cell types more suitable for imaging the axon morphology 459 with established axon-dependent functional readouts, such as wing neurons or olfactory receptor 460 neurons (ORNs) (Neukomm et al., 2017; Osterloh et al., 2012). 
464 With recent advances in the understanding of the Wallerian degeneration pathway, additional 465 regulators have been identified, including dSarm1 and Axed. Studies in Drosophila and mice suggest that dSarm 1 acts downstream of $\mathrm{Wld}^{\mathrm{S}}$ while Axed may be either downstream of dSarm 1

467 or involved in a separate pathway (Coleman and Höke, 2020; Neukomm et al., 2017; Osterloh et 468 al., 2012; Sambashivan and Freeman, 2021). In this study, we examined the roles of dSarm1 and 469 Axed in dendrites and found that their functions diverged from those of Wld $\mathrm{d}^{\mathrm{S}}$ during dendrite 470 development, caspase-3 induced dendrite degeneration, and the thermal nocifensive behavior.

Previous studies report that knockout of dSarm 1 specifically in c4da neurons does not

472 affect dendrite structure but can protect c4da neurons from injury and PS-induced dendrite 473 degeneration in wandering larvae during late larval development (Ji et al., 2021). Knockout of 474 Axed in c4da neurons partially affects dendrite degeneration induced by injury but does not alter 475 the degeneration in response to PS exposure (Ji et al., 2021). In this study, we found that 476 knockdown of dSarm1 but not Axed in c4da neurons led to longer dendrites during early dendrite 477 development and following caspase-3 induced dendrite degeneration. However, reduced dSarm1 478 expression in c4da neurons did not protect them against caspase-3 induced impairments in their 479 dendrite structure and neuronal functions later in the development during the wandering stage. At 480 the behavioral level, we found that knockdown of dSarm1 or Axed in c4da neurons of control 481 larvae with or without caspase-LOV impaired the thermal nocifensive behavior. Thus, dSarm1 and 482 Axed affect c4da neurons-mediated thermal nocifensive behavior of larvae without altering 483 dendrites of c4da neurons.

\section{Functions of dSarm1 and Axed in other Drosophila neurons and in mammalian neurons}

486 Apart from c4da neurons, roles of dSarm1 and Axed in dendrite morphology and neuronal 487 functions in other cell types have been described. Sarm1 knockdown in cultured hippocampal 488 neurons or in mice results in simplified dendrite structure instead of longer dendrites as we 489 observed in c4da neurons (Chen et al., 2011). In the mushroom body gamma neurons of the fly 490 central nervous system, dSarm1 and Axed mutations do not affect dendrite pruning (Neukomm et 491 al., 2017; Osterloh et al., 2012). For the behavioral functions, Sarm1 knockdown in mice causes 492 deficiency in associative memory, cognitive flexibility and social interactions (Lin and Hsueh, 493 2014), whereas flies containing dSarm1 or Axed mutant Johnston's organ (JO) clones can still 494 elicit JO neurons-mediated grooming behavior (Neukomm et al., 2017). It thus appears that the 
495 functions of these proteins may vary with their subcellular localization, the cell types, the time in

496 development, as well as the species.

\section{Advantages of the new model for caspase-3 induced neurodegeneration}

499 The range of dendrite degeneration and repair resulting from varying degrees of caspase-LOV

500 activation demonstrates the versatility of the photo-switchable caspase-3 system to induce

501 degeneration in Drosophila da neurons. This new model has several strengths. First, with photo-

502 switchable caspase-3, the timing and degree of degeneration can be controlled by adjusting the

503 length and intensity of illumination. The activation of caspase-LOV lasts for the duration of the

504 illumination and is reversible. In conjunction with the genetic tools available, we could induce

505 degeneration in specific cell types. Finer spatial control may be achieved by locally illuminating

506 certain areas of the cell viewed under the microscope or by targeting the photo-switchable caspase-

5073 with linked peptide sequences to specific subcellular compartments. Second, in contrast to laser

508 severing of dendrites, the photo-switchable caspase-3 allows for infliction of neuronal injury

509 systematically in a way that is considerably less labor intensive. It is thus amenable to screens of

510 genetic manipulations or pharmacological drug libraries to dissect the underlying cellular and

511 molecular mechanisms. Third, this model is physiologically relevant, given that caspase-3 plays a

512 role in the developmental pruning of axon and dendrite (Kuo et al., 2006; Williams et al., 2006;

513 Schoenmann et al., 2010) as well as axon degeneration initiated by trophic factor withdrawal

514 (Nikolaev et al., 2009; Schoenmann et al., 2010, Simon 2012). The discoveries made possible with

515 the photo-switchable caspase-3 system will therefore be likely to yield information about

516 physiologically relevant neurodegeneration that occurs during developmental pruning, trophic

517 factor withdrawal, and disease. Our model can complement the existing injury models, including

518 laser ablation-induced dendrite degeneration and PS-induced dendrite degeneration (Sapar et al.,

519 2018; Tao and Rolls, 2011) and provide an alternative route to study how to repair dendrites

520 following neurodegeneration. It is currently unclear whether neurons respond to different insults

521 the same way or whether insult-specific response pathways exist. In order to develop effective

522 therapies, it is important to investigate how neurons respond to different types of injuries.

\section{Possible improvements of the new model}


525 In this study, we set up a degeneration and repair model for larval sensory neurons. The repair

526 process identified in the larval sensory neurons could be a combination of developmental growth

527 and a repair response specific to caspase-3 induced degeneration. To focus on the contribution

528 from the repair process and to identify ways to re-establish the growth capacity of neurons, it is

529 desirable to extend the system to the adult sensory neurons. The adult sensory neurons reach

530 maturity around 3 days after eclosion and have stabilized dendrite structure throughout adulthood

531 (DeVault et al., 2018). Therefore, dendrite elongation or addition following caspase-3 induced

532 degeneration in adult fly would correspond to regeneration and repair.

In this proof-of-principle initial study, transgenes and RNAis are expressed before caspaseLOV activation, so the effects could be due to prevention of damage or repair of damage. Future improvements for better temporal control could make use of either a pharmacologically controlled gene switch system or the temperature-sensitive GAL80 repressor (Gal80ts) (Nicholson et al., 2008; Zeidler et al., 2004). Whereas we focused on cell autonomous factors in this study, we recognized there are likely non-cell autonomous contributions from epidermal cells and glial cells (DeVault et al., 2018; Liu and Jan, 2020; Song et al., 2012; Yadav et al., 2019; Yin et al., 2021). Future studies of dendrite degeneration at different stages of development as well as adulthood may shed light on strategies to prevent neurodegeneration, to diagnose neurodegeneration early, and to develop drugs promoting neural recovery from injury and diseases.

\section{METHODS}

\section{$545 \quad$ Fly stocks and genetics}

546 Animals were reared at $25^{\circ} \mathrm{C}$ or at $22^{\circ} \mathrm{C}$ for monitoring the dendrite degeneration and repair. The

547 fly strains used in this study were as follows: UAS-Wld ${ }^{\mathrm{S}}$ (a generous gift from Ashley Smart at 548 UCSF (Hoopfer et al., 2006)), Gal4 ${ }^{19-12}$ (Xiang et al., 2010), Gal42-21 (Grueber et al., 2003a), ppk-

549 Gal4 (Grueber et al., 2003b), ppk-CD4-tdGFP (Han et al., 2011), UAS-caspase-LOV (BL76355, 550 a generous gift from Ashley Smart at UCSF), UAS-tdTomato (Han et al., 2011), UAS-mIFP-T2A$551 \mathrm{HO} 1$ (attp40 on $2^{\text {nd }}$ chromosome used in this study. a generous gift from Xiaokun Shu, UCSF), 552 UAS-luciferase (BL35788, control RNAi for the TRiP lines) UAS-dSarm1-RNAi (BL 63525), 553 UAS-Axed-RNAi (BL 62989). The RNAi lines we used in the study are all VALIUM20-series 554 TRiP RNAi fly stocks that produce short hairpin RNAs (shRNAs) and give stronger knockdown 555 efficiency then VALIUM10-series TRiP RNAi flies (Ni et al., 2011). The tester lines for RNA 
556 interference (RNAi) or overexpression experiments was ppk-gal4, ppk-CD4-tdGFP; UAS-

557 caspase-LOV. RNAi or overexpression experiments were performed by crossing the tester lines to 558 the variety of transgenic fly strains. To control for caspase-LOV expression dosage in different 559 genotypes, we used UAS-mIFP-T2A-HO1 (wild-type, $\mathrm{w}^{1118}$ ) as control for Wld ${ }^{\mathrm{S}}$ experiments and 560 UAS-luciferase (yv flies) as control for RNAi experiments. We found slight differences for 561 thermal nocifensive behavior in genotypes, so we used different fly strains as controls for Wld 562 and RNAi lines.

\section{Illumination box with LED strips}

565 We collect eggs laid in the dark for $2 \mathrm{~h}$ and kept them in the dark at $25^{\circ} \mathrm{C}$ until illuminated at $48 \mathrm{~h}$ 566 after egg laying (AEL). To activate the photo-switchable caspase-3, freely moving larvae were picked and transferred the transparent agar plates with a thin layer of yeast. Larvae were moved

568 back to yeasted grape plate and kept in the dark at $22^{\circ} \mathrm{C}$ after different durations of blue LED 569 illumination. Lower raising temperature to $22^{\circ} \mathrm{C}$ can delay development and increase the temporal resolution of the repair process following caspase-3 activation. To avoid lights, grape juice plates are store in $10 \mathrm{~mm}$ petri dishes wrapped with foil. A homemade $40 \mathrm{~cm}$ x $10 \mathrm{~cm}$ x $15 \mathrm{~cm}$ carbon

572 box was used to shield larvae from ambient light and to house three $10 \mathrm{~cm}$ long and $8 \mathrm{~mm}$ wide 573 Blue 3528 LED strip, (peak at 460nm, Environmental Lights) stick on the ceiling of the box in 574 parallel and connected by wires. LED strips are wired to a connector with DC jack (Environmental 575 Lights) and then a LED Power Supply Adapter (HitLights). The power of the light $15 \mathrm{~cm}$ away from the LED strips, where larvae were kept, is $0.91 \mathrm{~mW} / \mathrm{cm}^{2}$.

\section{In vivo time lapse imaging}

579 Live imaging was performed as described (Emoto et al., 2006; Parrish et al., 2007). Larvae were anesthetized with diethyl-ether for 5-8 minutes (Acros Organics) before mounted in glycerol on top of a thin patch of agarose. After images were acquired using a Leica SP5 microscope with a 20X oil objective (NA 0.75), larva was returned to yeasted grape juice agar plates or sacrificed if this is the end of imaging timepoints. Sum slices for Z-projection were generated using ImageJ software and used for dendrite structure prediction as described later.

To visualize neurons, c4da ddaC neurons were labeled by expressing UAS-CD4-tdTOM 
587

visualized through mCD4-tdTOM driven by Gal4 ${ }^{2-21}$. C3da ddaF neuron with UAS-CD4-tdTOM driven by the GAL4 ${ }^{19-21}$ along with Repo-Gal80 to eliminate the expression in glial cells (Awasaki et al., 2008; Xiang et al., 2010)

\section{Deep learning based-automatic dendrite structure prediction}

We utilized two methods to segment the dendrite structures of the da neurons for morphological quantification. For ddaE, clda neurons, and ddac, c4da neurons, in Figure 1, we reconstructed individual neurons using Vaa3D-Neuron 2.0: 3D neuron paint and tracing function in Vaa3D (http://vaa3d.org/) with manual correction and validation of the tracing (Peng et al., 2010).

For the rest of ddaC neurons in this study, we established a U-Net based deep learning model for automatic dendrite structure segmentation which produces segmentation maps with pixel intensity representing the probability of dendrite structure. We followed the U-Net architecture specified in the original study (Ronneberger et al., 2015) with modifying the channel number of the final segmentation map from 2 to 1 since we only predicted dendrite structure versus background. Each training data consisted of a maximum intensity Z-projection image of one neuron manually cropped by drawing a ROI, paired with the manually segmented dendrite structure (mask) generated using the plugin, "simple neurite tracer", in ImageJ. In total, we generated 29 sets of image-mask pairs for training and 8 sets for validation with datasets generated in-house. Two data augmentation strategies were used to increase the model robustness. First, an area of 512x512 pixels was randomly cropped from each input 1024x1024 training image and the associated mask. Then the cropped image and mask were randomly flipped horizontally and vertically with probability 0.5 . We used the sum of binary cross-entropy and Dice loss (defined as 1 - Dice coefficient) as the loss function and trained the model with Adam optimizer at learning rate 1e-4 for 500 epochs. The best model evaluated by Dice loss using the validation dataset was chosen for the downstream analysis. Our best model achieved the Dice loss at 0.13 and 0.16 for training and validation datasets, respectively.

A threshold of 0.5 was used to binarize segmentation maps generated by the model. We found high correlation $\left(\mathrm{R}^{2}=0.98\right)$ in total dendrite length of larval neurons between modelpredicted segmentation and manual reconstruction, while tip numbers only showed moderate correlation $\left(\mathrm{R}^{2}=0.45\right)$. This was because tip number was more sensitive to the discontinuity and small fragments occasionally found in model-predicted segmentation masks. Therefore, we 
618 included a 3-step post-processing procedure to exclude small fragments and reduce the

619 discontinuity in the segmented dendrite structure. First, small objects with area less than 10 pixels

620 were discarded. Second, dilation with a cross-shaped structuring element (connectivity=1) was

621 used to fill in the gaps. Finally, skeletonization using the skeletonize function from Python scikit-

622 image package was applied to obtain the final segmentation for the downstream morphology

623 quantification. With post-processing to fill in gaps and remove small fragments, we observed a

624 dramatic increase in the correlation of tip numbers $\left(\mathrm{R}^{2}=0.97\right)$ and a slight increase for total

625 dendrite length $\left(\mathrm{R}^{2}=0.99\right)$.

This system can be applied to predict the structures of other type of neurons either using

627 the exiting models or retain models with new set of training datasets. One limitation is to separate

628 the individual neurons at the manual ROI selection step. For example, the Gal4 ${ }^{19-12}$ and Gal4-21

629 drivers sometimes have weak expression in surrounding neurons which is hard to separate. When

630 the neurons are also well-marked by fluorescence proteins, they can be recognized by the

631 prediction model and included as part of the c1da neurons which introduce false positive errors.

632 Therefore, we did not use the model for the c1da neurons.

633

\section{Quantification of dendrite structure}

635 With the prediction model described above along with the post-processing python code, we can

636 obtain the total dendrite length, total dendrite tip numbers and skeletal images of predicted dendrite

637 structures. Using the skeletal images, we performed Sholl analysis of dendrite branches to

638 determine the complexity of the dendrite structure. The crossing continuous circles were separated

639 by $0.76 \mu \mathrm{m}$ on either manually traced or predicted dendrite arbors. To determine percentage of

640 territory covered, we measured the area of dendrite arbor of neuron of interest covered and divided

641 it to total area of the hemisegment of the body wall. The territory covered is measured using ROI

642 selection tools in ImageJ. We defined a cell as "survived" if the average dendrite length (total

643 dendrite length/total tip numbers) over $10 \mu \mathrm{m}$ for c4da neurons. For c3da and c1da neurons, we

644 identified neurons with more than 2 dendrite tips (more than one dendrite branch) as survived. To

645 reduce the batch-by-batch variations, we normalized the quantifications to the controls for each

646 batch before combining all data. For comparison between different conditions, the number was

647 normalized to the averaged number in dark (control). The results are normalized to the controls for 648 each set of experiments before combining. 


\section{Thermal nocifensive behavior}

651 For thermo-nociception using a local hot probe, a custom-built thermo-couple device was used to

652 keep the applied temperature constantly at 42 or $46{ }^{\circ} \mathrm{C}$ as desired. Stage and density-controlled

6533 3rd instar wandering stage larvae were used. Freely moving larvae were touched with the hot probe

654 on mid-abdominal segments until the execution of nociceptive rolling avoidance behavior.

655 Animals were monitored under cell phone camera (Nokia 6.1) and the time it takes to initiate the

656 rolling behavior for high temperature were counted with in $20 \mathrm{~s}$. The animals that take longer than

$65720 \mathrm{~s}$ to response were classified as no responders. The percentages of no responder were calculated

658 by dividing numbers of no responders by numbers of total tested animals. Each genotype was

659 tested multiple times on different days and data from all trials was combined.

660

661 Software

662 The code used for deep learning based automatic dendrite structure prediction is written in 663 python/TensorFlow. We trained our model on a Quadro P5000 GPU with 16 GB RAM in a Dell 664 Precision 7920 Tower with Dual Intel Xeon Gold 6136 CPUs (3.0/3.7GHz), having 12 cores and 665128 GB RAM. The operating system was Windows 10. We have tested our system on Mac and 666 Windows operating system. The software package, training and example testing images are 667 available on the GitHub repository (https://github.com/chienhsiang/dendrite_U-Net).

\section{Statistical tests}

670 All data are presented as mean \pm standard error of the mean (SEM) based on at least three 671 independent experiments. Data are considered significantly different when $\mathrm{p}$ values are less than 672 0.05. Student's t test was used for comparisons of two groups. One-way ANOVA with Tukey's 673 post hoc test was used for comparisons of multiple groups. The Kruskal-Wallis rank sum test with 674 Dunn's post hoc test further adjusted by the Benjamini-Hochberg FDR method was used for 675 multiple comparisons of nonparametric samples. Statistics analysis was performed and prepared 676 using JASP (Version 0.14). All samples were prepared and analyzed in parallel.

\section{ACKNOWLEDGMENTS}


679 We would like to thank members of the Jan Lab for helpful discussions. We want to thank Jacob

680 Jaszczak, Caitlin O'Brien, Liying Li, and Ashely Smart for critical reading and suggestions on the 681 manuscript. We are grateful for Ashley Smart and Xiaokun Shu at UCSF for kindly sharing fly

682 stocks with us. We are grateful for Caitlin O'Brien for providing the training datasets. Research 683 reported in this publication was supported by the National Institute of Neurological Disorders and 684 Stroke (R35NS097227 to YNJ). Yuh-Nung Jan and Lily Y. Jan are investigators at the Howard 685 Hughes Medical Institute.

\section{COMPETING INTERESTS}

688 The authors declare no competing interests.

\section{REFERENCES}

Awasaki T, Lai S-L, Ito K, Lee T. 2008. Organization and Postembryonic Development of Glial Cells in the Adult Central Brain of Drosophila. J Neurosci 28:13742-13753. doi:10.1523/JNEUROSCI.4844-08.2008

Babcock DT, Landry C, Galko MJ. 2009. Cytokine signaling mediates UV-induced nociceptive sensitization in Drosophila larvae. Curr Biol CB 19:799-806. doi:10.1016/j.cub.2009.03.062

Chen C-Y, Lin C-W, Chang C-Y, Jiang S-T, Hsueh Y-P. 2011. Sarm1, a negative regulator of innate immunity, interacts with syndecan-2 and regulates neuronal morphology. J Cell Biol 193:769-784. doi:10.1083/jcb.201008050

Coleman MP, Höke A. 2020. Programmed axon degeneration: from mouse to mechanism to medicine. Nat Rev Neurosci 21:183-196. doi:10.1038/s41583-020-0269-3

Cotman CW, Su JH. 1996. Mechanisms of neuronal death in Alzheimer's disease. Brain Pathol 6:493-506.

DeVault L, Li T, Izabel S, Thompson-Peer KL, Jan LY, Jan YN. 2018. Dendrite regeneration of adult Drosophila sensory neurons diminishes with aging and is inhibited by epidermalderived matrix metalloproteinase 2. Genes Dev 32:402-414. doi:10.1101/gad.308270.117

Eldadah BA, Faden AI. 2000. Caspase pathways, neuronal apoptosis, and CNS injury. $J$ Neurotrauma 17:811-29. doi:10.1089/neu.2000.17.811

Erturk A, Wang Y, Sheng M. 2014. Local pruning of dendrites and spines by caspase-3-dependent and proteasome-limited mechanisms. $J$ Neurosci 34:1672-88. doi:10.1523/JNEUROSCI.3121-13.2014

Gerdts J, Summers DW, Sasaki Y, DiAntonio A, Milbrandt J. 2013. Sarm1-mediated axon degeneration requires both SAM and TIR interactions. J Neurosci Off J Soc Neurosci 33:13569-13580. doi:10.1523/JNEUROSCI.1197-13.2013

Gorczyca DA, Younger S, Meltzer S, Kim SE, Cheng L, Song W, Lee HY, Jan LY, Jan YN. 2014. Identification of Ppk26, a DEG/ENaC Channel Functioning with Ppk1 in a Mutually Dependent Manner to Guide Locomotion Behavior in Drosophila. Cell Rep 9:1446-58. doi:10.1016/j.celrep.2014.10.034 
Grueber WB, Jan LY, Jan YN. 2003a. Different levels of the homeodomain protein cut regulate distinct dendrite branching patterns of Drosophila multidendritic neurons. Cell 112:80518. doi:10.1016/s0092-8674(03)00160-0

Grueber WB, Jan LY, Jan YN. 2002. Tiling of the Drosophila epidermis by multidendritic sensory neurons. Development 129:2867-78.

Grueber WB, Ye B, Moore AW, Jan LY, Jan YN. 2003b. Dendrites of distinct classes of Drosophila sensory neurons show different capacities for homotypic repulsion. Curr Biol 13:618-26. doi:10.1016/s0960-9822(03)00207-0

Han C, Jan LY, Jan Y-N. 2011. Enhancer-driven membrane markers for analysis of nonautonomous mechanisms reveal neuron-glia interactions in Drosophila. Proc Natl Acad Sci U S A 108:9673-9678. doi:10.1073/pnas.1106386108

Hertzler JI, Simonovitch SI, Albertson RM, Weiner AT, Nye DMR, Rolls MM. 2020. Kinetochore proteins suppress neuronal microtubule dynamics and promote dendrite regeneration. $\mathrm{Mol}$ Biol Cell 31:2125-2138. doi:10.1091/mbc.E20-04-0237-T

Honjo K, Mauthner SE, Wang Y, Skene JHP, Tracey WD. 2016. Nociceptor-enriched genes required for normal thermal nociception. Cell Rep 16:295-303. doi:10.1016/j.celrep.2016.06.003

Hoopfer ED, McLaughlin T, Watts RJ, Schuldiner O, O’Leary DDM, Luo L. 2006. Wlds protection distinguishes axon degeneration following injury from naturally occurring developmental pruning. Neuron 50:883-895. doi:10.1016/j.neuron.2006.05.013

Hwang RY, Stearns NA, Tracey WD. 2012. The ankyrin repeat domain of the TRPA protein painless is important for thermal nociception but not mechanical nociception. PLoS One 7:e30090. doi:10.1371/journal.pone.0030090

Hwang RY, Zhong L, Xu Y, Johnson T, Zhang F, Deisseroth K, Tracey WD. 2007. Nociceptive neurons protect Drosophila larvae from parasitoid wasps. Curr Biol CB 17:2105-2116. doi:10.1016/j.cub.2007.11.029

Jan Y-N, Jan LY. 2010. Branching out: mechanisms of dendritic arborization. Nat Rev Neurosci 11:316-328. doi:10.1038/nrn2836

Ji H, Sapar ML, Sarkar A, Wang B, Han C. 2021. Phagocytosis and self-destruction execute dendrite degeneration of Drosophila sensory neurons at distinct levels of NAD+ reduction. bioRxiv 2020.06.26.173245. doi:10.1101/2020.06.26.173245

Kanaoka Y, Skibbe H, Hayashi Y, Uemura T, Hattori Y. 2019. DeTerm: Software for automatic detection of neuronal dendritic branch terminals via an artificial neural network. Genes Cells Devoted Mol Cell Mech 24:464-472. doi:10.1111/gtc.12700

Khatri N, Gilbert JP, Huo Y, Sharaflari R, Nee M, Qiao H, Man HY. 2018. The Autism Protein Ube3A/E6AP Remodels Neuronal Dendritic Arborization via Caspase-Dependent Microtubule Destabilization. J Neurosci 38:363-378. doi:10.1523/JNEUROSCI.151117.2017

Kim SE, Coste B, Chadha A, Cook B, Patapoutian A. 2012. The role of Drosophila Piezo in mechanical nociception. Nature 483:209-212. doi:10.1038/nature10801

Kitatani Y, Tezuka A, Hasegawa E, Yanagi S, Togashi K, Tsuji M, Kondo S, Parrish JZ, Emoto K. 2020. Drosophila miR-87 promotes dendrite regeneration by targeting the transcriptional repressor Tramtrack69. PLoS Genet 16:e1008942. doi:10.1371/journal.pgen.1008942

Kulkarni VA, Firestein BL. 2012. The dendritic tree and brain disorders. Mol Cell Neurosci 50:1020. doi:10.1016/j.men.2012.03.005 
Kuo CT, Zhu S, Younger S, Jan LY, Jan YN. 2006. Identification of E2/E3 ubiquitinating enzymes and caspase activity regulating Drosophila sensory neuron dendrite pruning. Neuron 51:283-90. doi:10.1016/j.neuron.2006.07.014

Kweon JH, Kim S, Lee SB. 2017. The cellular basis of dendrite pathology in neurodegenerative diseases. BMB Rep 50:5-11.

Lin C-W, Hsueh Y-P. 2014. Sarm1, a neuronal inflammatory regulator, controls social interaction, associative memory and cognitive flexibility in mice. Brain Behav Immun 37:142-151. doi:10.1016/j.bbi.2013.12.002

Liu H-H, Jan Y-N. 2020. Mechanisms of neurite repair. Curr Opin Neurobiol 63:53-58. doi:10.1016/j.conb.2020.02.010

Lunn ER, Perry VH, Brown MC, Rosen H, Gordon S. 1989. Absence of Wallerian Degeneration does not Hinder Regeneration in Peripheral Nerve. Eur $J$ Neurosci 1:27-33. doi:10.1111/j.1460-9568.1989.tb00771.x

MacDonald JM, Beach MG, Porpiglia E, Sheehan AE, Watts RJ, Freeman MR. 2006. The Drosophila cell corpse engulfment receptor Draper mediates glial clearance of severed axons. Neuron 50:869-881. doi:10.1016/j.neuron.2006.04.028

Mulherkar S, Firozi K, Huang W, Uddin MD, Grill RJ, Costa-Mattioli M, Robertson C, Tolias KF. 2017. RhoA-ROCK Inhibition Reverses Synaptic Remodeling and Motor and Cognitive Deficits Caused by Traumatic Brain Injury. Sci Rep 7:10689. doi:10.1038/s41598-01711113-3

Nagel J, Delandre C, Zhang Y, Förstner F, Moore AW, Tavosanis G. 2012. Fascin controls neuronal class-specific dendrite arbor morphology. Dev Camb Engl 139:2999-3009. doi:10.1242/dev.077800

Neukomm LJ, Burdett TC, Seeds AM, Hampel S, Coutinho-Budd JC, Farley JE, Wong J, Karadeniz YB, Osterloh JM, Sheehan AE, Freeman MR. 2017. Axon Death Pathways Converge on Axundead to Promote Functional and Structural Axon Disassembly. Neuron 95:78-91.e5. doi:10.1016/j.neuron.2017.06.031

Ni J-Q, Zhou R, Czech B, Liu L-P, Holderbaum L, Yang-Zhou D, Shim H-S, Tao R, Handler D, Karpowicz P, Binari R, Booker M, Brennecke J, Perkins LA, Hannon GJ, Perrimon N. 2011. A genome-scale shRNA resource for transgenic RNAi in Drosophila. Nat Methods 8:405-407. doi:10.1038/nmeth.1592

Nicholson L, Singh GK, Osterwalder T, Roman GW, Davis RL, Keshishian H. 2008. Spatial and temporal control of gene expression in Drosophila using the inducible GeneSwitch GAL4 system. I. Screen for larval nervous system drivers. Genetics 178:215-34. doi:10.1534/genetics.107.081968

Nye DMR, Albertson RM, Weiner AT, Hertzler JI, Shorey M, Goberdhan DCI, Wilson C, Janes KA, Rolls MM. 2020. The receptor tyrosine kinase Ror is required for dendrite regeneration in Drosophila neurons. PLoS Biol 18:e3000657. doi:10.1371/journal.pbio.3000657

Osterloh JM, Yang J, Rooney TM, Fox AN, Adalbert R, Powell EH, Sheehan AE, Avery MA, Hackett R, Logan MA, MacDonald JM, Ziegenfuss JS, Milde S, Hou YJ, Nathan C, Ding A, Brown RH, Conforti L, Coleman M, Tessier-Lavigne M, Zuchner S, Freeman MR. 2012. $\mathrm{dSarm} / \mathrm{Sarm} 1$ is required for activation of an injury-induced axon death pathway. Science 337:481-4. doi:10.1126/science.1223899 
Parrish JZ, Xu P, Kim CC, Jan LY, Jan YN. 2009. The microRNA bantam functions in epithelial cells to regulate scaling growth of dendrite arbors in drosophila sensory neurons. Neuron 63:788-802. doi:10.1016/j.neuron.2009.08.006

Penzes P, Cahill ME, Jones KA, VanLeeuwen J-E, Woolfrey KM. 2011. Dendritic spine pathology in neuropsychiatric disorders. Nat Neurosci 14:285-293. doi:10.1038/nn.2741

Ronneberger O, Fischer P, Brox T. 2015. U-Net: Convolutional Networks for Biomedical Image Segmentation. ArXiv150504597 Cs.

Sambashivan S, Freeman MR. 2021. SARM1 signaling mechanisms in the injured nervous system. Curr Opin Neurobiol 69:247-255. doi:10.1016/j.conb.2021.05.004

Sapar ML, Ji H, Wang B, Poe AR, Dubey K, Ren X, Ni J-Q, Han C. 2018. Phosphatidylserine Externalization Results from and Causes Neurite Degeneration in Drosophila. Cell Rep 24:2273-2286. doi:10.1016/j.celrep.2018.07.095

Schoenmann Z, Assa-Kunik E, Tiomny S, Minis A, Haklai-Topper L, Arama E, Yaron A. 2010. Axonal Degeneration Is Regulated by the Apoptotic Machinery or a NAD+-Sensitive Pathway in Insects and Mammals. $J$ Neurosci 30:6375-6386. doi:10.1523/JNEUROSCI.0922-10.2010

Shimono K, Fujimoto A, Tsuyama T, Yamamoto-Kochi M, Sato M, Hattori Y, Sugimura K, Usui T, Kimura K, Uemura T. 2009. Multidendritic sensory neurons in the adult Drosophila abdomen: origins, dendritic morphology, and segment- and age-dependent programmed cell death. Neural Dev 4:37. doi:10.1186/1749-8104-4-37

Simon DJ, Pitts J, Hertz NT, Yang J, Yamagishi Y, Olsen O, Tesic Mark M, Molina H, TessierLavigne M. 2016. Axon Degeneration Gated by Retrograde Activation of Somatic Proapoptotic Signaling. Cell 164:1031-45. doi:10.1016/j.cell.2016.01.032

Smart AD, Pache RA, Thomsen ND, Kortemme T, Davis GW, Wells JA. 2017. Engineering a light-activated caspase-3 for precise ablation of neurons in vivo. Proc Natl Acad Sci U A 114:E8174-E8183. doi:10.1073/pnas.1705064114

Song Y, Ori-McKenney KM, Zheng Y, Han C, Jan LY, Jan YN. 2012. Regeneration of Drosophila sensory neuron axons and dendrites is regulated by the Akt pathway involving Pten and microRNA bantam. Genes Dev 26:1612-25. doi:10.1101/gad.193243.112

Stone MC, Albertson RM, Chen L, Rolls MM. 2014. Dendrite injury triggers DLK-independent regeneration. Cell Rep 6:247-53. doi:10.1016/j.celrep.2013.12.022

Sugimura K, Yamamoto M, Niwa R, Satoh D, Goto S, Taniguchi M, Hayashi S, Uemura T. 2003. Distinct developmental modes and lesion-induced reactions of dendrites of two classes of Drosophila sensory neurons. J Neurosci 23:3752-60.

Tao J, Rolls MM. 2011. Dendrites have a rapid program of injury-induced degeneration that is molecularly distinct from developmental pruning. $J$ Neurosci 31:5398-5405. doi:10.1523/JNEUROSCI.3826-10.2011

Thompson-Peer KL, DeVault L, Li T, Jan LY, Jan YN. 2016. In vivo dendrite regeneration after injury is different from dendrite development. Genes Dev 30:1776-89. doi:10.1101/gad.282848.116

Tsubouchi A, Caldwell JC, Tracey WD. 2012. Dendritic filopodia, Ripped Pocket, NOMPC, and NMDARs contribute to the sense of touch in Drosophila larvae. Curr Biol CB 22:21242134. doi:10.1016/j.cub.2012.09.019

Williams DW, Kondo S, Krzyzanowska A, Hiromi Y, Truman JW. 2006. Local caspase activity directs engulfment of dendrites during pruning. Nat Neurosci 9:1234-6. doi:10.1038/nn1774 
Williams DW, Truman JW. 2005. Cellular mechanisms of dendrite pruning in Drosophila: insights from in vivo time-lapse of remodeling dendritic arborizing sensory neurons. Dev Camb Engl 132:3631-3642. doi:10.1242/dev.01928

Xiang Y, Yuan Q, Vogt N, Looger LL, Jan LY, Jan YN. 2010. Light-avoidance-mediating photoreceptors tile the Drosophila larval body wall. Nature 468:921-6. doi:10.1038/nature09576

Xiong Y, Mahmood A, Chopp M. 2019. Remodeling dendritic spines for treatment of traumatic brain injury. Neural Regen Res 14:1477-1480. doi:10.4103/1673-5374.255957

Yadav S, Younger SH, Zhang L, Thompson-Peer KL, Li T, Jan LY, Jan YN. 2019. Glial ensheathment of the somatodendritic compartment regulates sensory neuron structure and activity. Proc Natl Acad Sci U S A 116:5126-5134. doi:10.1073/pnas.1814456116

Yan Z, Zhang W, He Y, Gorczyca D, Xiang Y, Cheng LE, Meltzer S, Jan LY, Jan YN. 2013. Drosophila NOMPC is a mechanotransduction channel subunit for gentle-touch sensation. Nature 493:221-225. doi:10.1038/nature11685

Yin C, Peterman E, Rasmussen JP, Parrish JZ. 2021. Transparent Touch: Insights From Model Systems on Epidermal Control of Somatosensory Innervation. Front Cell Neurosci 15:680345. doi:10.3389/fncel.2021.680345

Zeidler MP, Tan C, Bellaiche Y, Cherry S, Häder S, Gayko U, Perrimon N. 2004. Temperaturesensitive control of protein activity by conditionally splicing inteins. Nat Biotechnol 22:871-876. doi:10.1038/nbt979

Zhong L, Hwang RY, Tracey WD. 2010. Pickpocket is a DEG/ENaC protein required for mechanical nociception in Drosophila larvae. Curr Biol 20:429-34. doi:10.1016/j.cub.2009.12.057

Zou KH, Warfield SK, Bharatha A, Tempany CMC, Kaus MR, Haker SJ, Wells WM, Jolesz FA, Kikinis R. 2004. Statistical validation of image segmentation quality based on a spatial overlap index. Acad Radiol 11:178-189. doi:10.1016/s1076-6332(03)00671-8 



B


radius from soma (um)

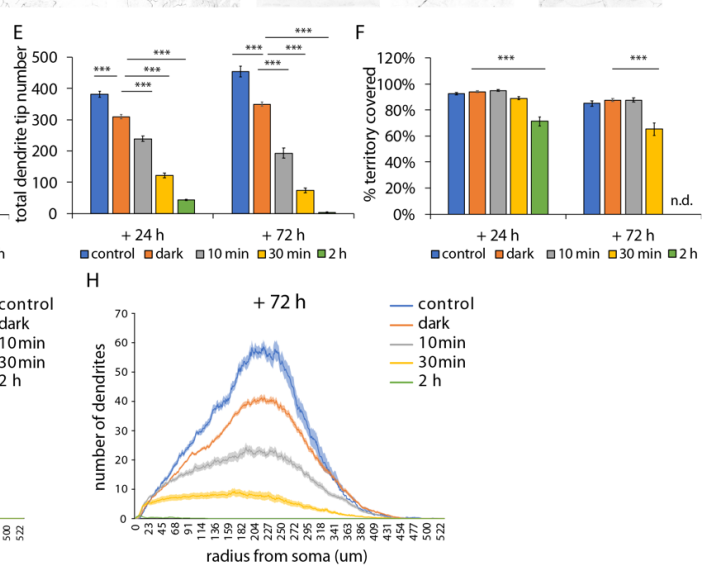

Figure 1. Transient caspase-LOV activation initiates dendrite degeneration followed by repair in c4da neurons. (A) Protocol to illuminate and image larval c4da neurons expressing just UAS-tdTOM (control) or UAS-tdTOM and UAS-caspase-LOV (dark, 10 min-24 h) using ppk-GAL4. These neurons were labeled with tdTOM for visualization. Larvae were kept in the dark all the time (control, dark) or kept in the dark and illuminated at $48 \mathrm{~h}$ after egg laying for $10 \mathrm{~min}-24 \mathrm{~h}$. The same neurons were imaged twice at $24 \mathrm{~h}$ and $72 \mathrm{~h}$ following illumination. (B) Representative images of c4da neurons from larva without caspase-LOV and kept in the dark (control), with caspase-LOV and kept in the dark (dark), or with caspaseLOV and illuminated for different durations (10 min-24 h). Neurons were imaged at $24 \mathrm{~h}(+24 \mathrm{~h}$, top row) and $72 \mathrm{~h}(+72 \mathrm{~h}$, bottom row) after illumination started. (C) Survival rates of c4da neurons expressing caspase-LOV decrease when illumination is extended. Survival of neurons was counted $72 \mathrm{~h}$ after illumination. (D-F) Quantifications of dendrite structures of survived c4da neurons following caspase-LOV activation, including total dendrite length (D), total dendrite tip numbers (E), and percentage of territory covered (F). The skeletal dendrite structures were predicted by in-house built deep learning models. The quantifications were carried out using a python script. (G-H) Sholl analysis of dendrite complexity $24 \mathrm{~h}(\mathrm{G})$ and $72 \mathrm{~h}(\mathrm{H})$ after illumination. The complexity of the dendrite structure, quantified as numbers of dendrites crossing continuous circles originated from the soma and represented by the total area under the curve, decreases with caspase-LOV expression and progresses as illumination extends. All conditions are significantly different from each other $(\mathrm{p}<0.01)$. Scale bars $=100 \mu \mathrm{m} . * \mathrm{p}<0.05, * * \mathrm{p}<0.01, * * * \mathrm{p}<0.001$, Kruskal-Wallis rank sum test with Dunn's post hoc test further adjusted by the Benjamini-Hochberg FDR method for multiple independent samples (C); one-way ANOVA with Tukey's post hoc test for multiple comparisons in $(\mathrm{D}-\mathrm{H})$. Error bars represent \pm SEM $(\mathrm{C}-\mathrm{F})$ or in shaded area $(\mathrm{G}-\mathrm{H}) . \mathrm{n}=14-55$ neurons for each experimental condition and timepoint. 

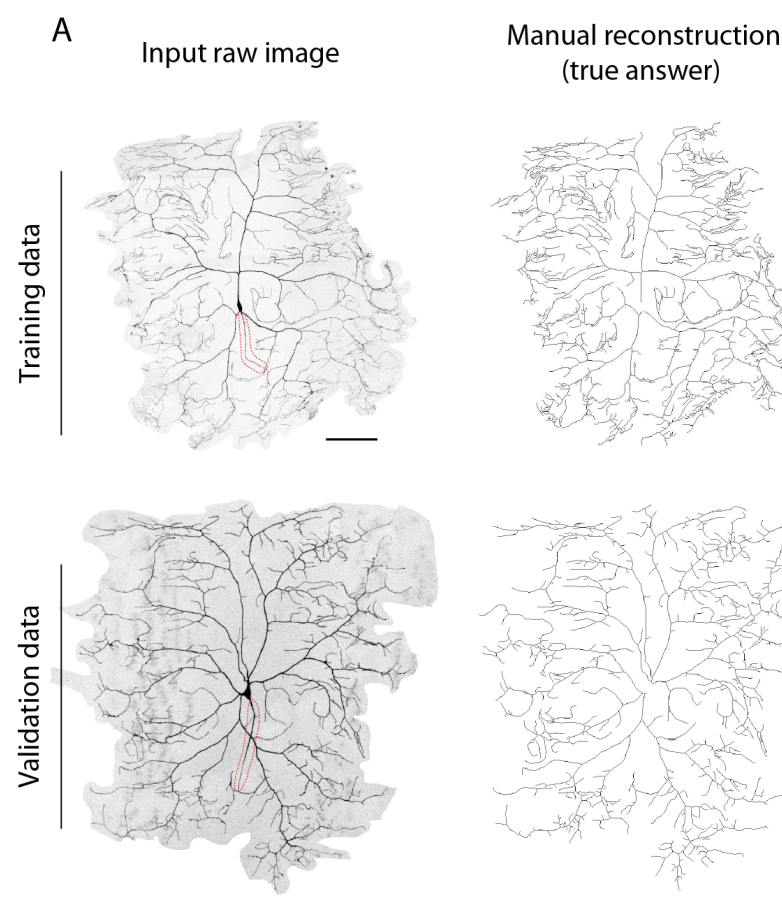

B


(true answer)



Model prediction
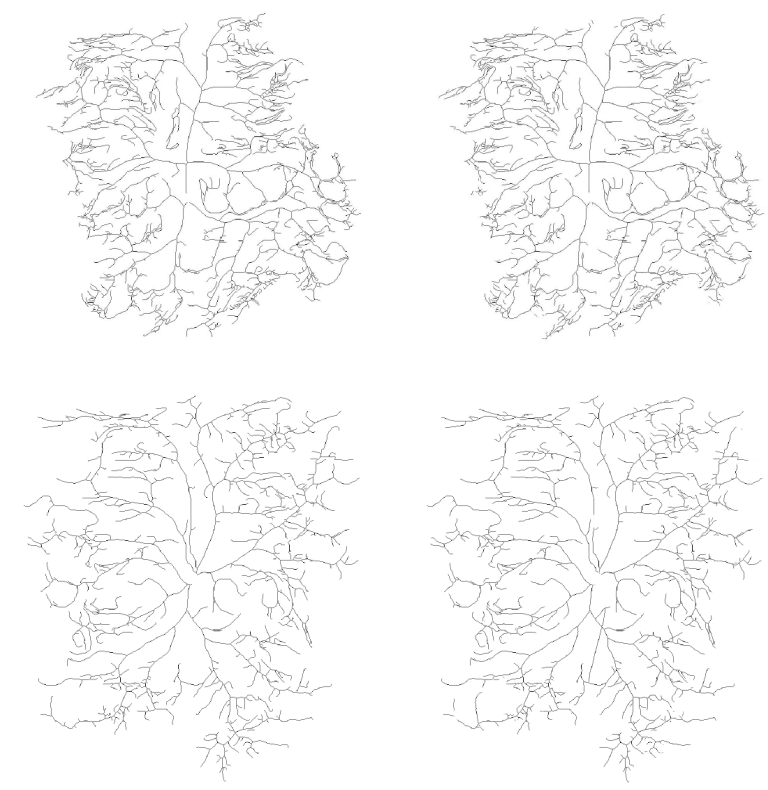

C

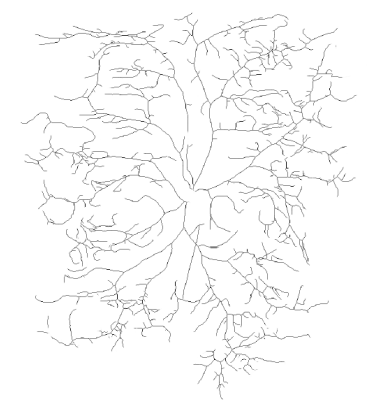



Red: model prediction
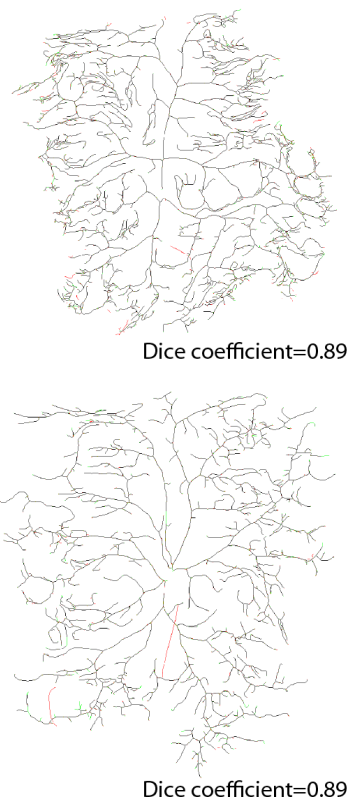

Total dendrite tip number

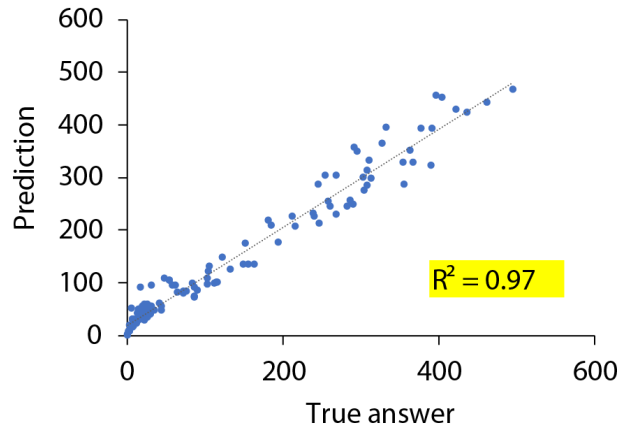

924
Figure 1 - figure supplement 1. Deep learning-based automatic dendrite structure prediction. (A) Our in-house trained deep learning-based model performed well in dendrite segmentation. In the top row are images of a representative neuron from the training dataset and the bottom row is a neuron from the validation dataset (novel neurons for the model). The first column contains input Z-projection image of neurons manually cropped by drawing a ROI. Images in the second column are manually segmented dendrite structure (true answer) from ImageJ plugin, "simple neurite tracer". Our model predictions are in the third column. The last column has overlay images from true answer and model prediction. The model reliably recognized most of the arbors as human as most of the dendrites are matched (marked in black) with few distal dim dendrites omitted by the model and only shown in the true answer (green) or only recognized by the model (red). Our model did not differentiate between axons and dendrites and sometimes counts the axon (circled with red dash line in the first column) as one of the dendrites ( 7 out of 37 neurons in the training and validation dataset). (B-C) Relationships between manual reconstruction (true answer) and the deep learning model (prediction) for total dendrite length and total dendrite tip number. After postprocessing, our prediction model achieved 0.99 for $\mathrm{R}^{2}$ of total dendrite length (B) and 0.97 for $\mathrm{R}^{2}$ of tip numbers $(\mathrm{C})$. Scale bars $=100 \mu \mathrm{m} . \mathrm{n}=160$ neurons. 

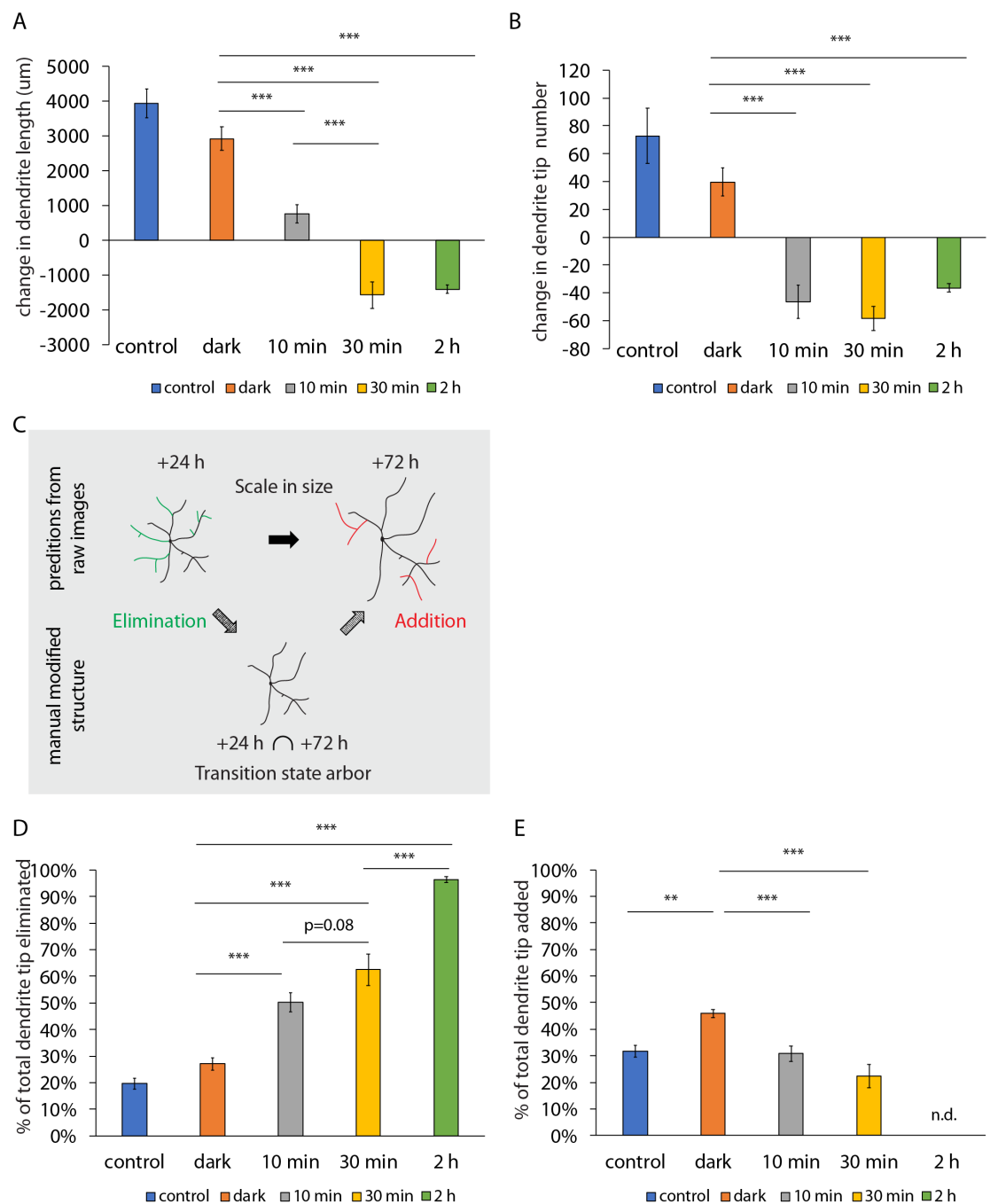

Figure 2. Dendrite addition and elimination occurs simultaneously during the repair process. (A-B)

Quantifications of changes in dendrite length (A) and dendrite tip numbers (B) of c4da neurons during the $24 \mathrm{~h}$ to $72 \mathrm{~h}$ time period after caspase-LOV activation. C4da neurons expressing caspase-LOV decrease growth in dendrite length and dendrite tip numbers as illumination is extended (C) Illustration of elimination and addition of dendrites happened over the degeneration and repair process. (D-E) Quantifications for the percentage of eliminated (D) and added (E) dendrite tips over the $24 \mathrm{~h}$ to $72 \mathrm{~h}$ time period following caspase-LOV activation. The percentage of tips eliminated increases with longer illumination while the percentage of tips added decreases. ${ }^{*} \mathrm{p}<0.05,{ }^{* *} \mathrm{p}<0.01,{ }^{* * *} \mathrm{p}<0.001$, one-way ANOVA with Tukey's post hoc test for multiple comparison in (A-B, D-E). Error bars represent \pm SEM. $n=19-23$ neurons for each experimental condition and timepoint. 


A $\frac{\text { no caspase-LOV }}{\text { control }} \stackrel{\text { caspase-LOV }}{\text { dark } 30 \mathrm{~min} \quad 2 \mathrm{~h}}$ B
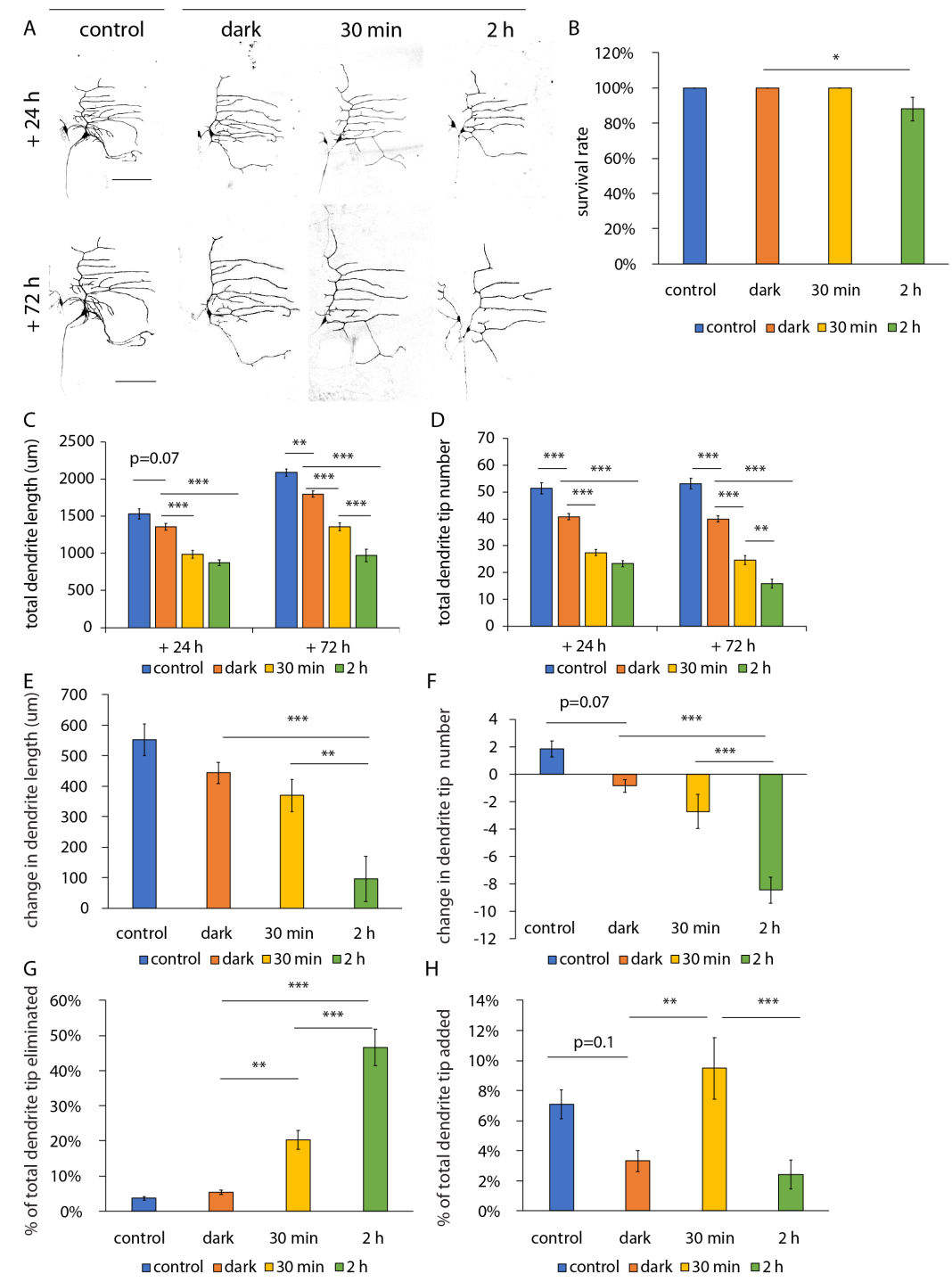

Figure 3. Class I ddaE neurons can sustain mild caspase-LOV activation and repair by adding new branches. (A) Representative images of c1da neurons expressing just UAS-tdTOM (control) or UAStdTOM and UAS-caspase-LOV (dark, $30 \mathrm{~min}, 2 \mathrm{~h}$ ) driven by ppk ${ }^{2-21}$-GAL4. Larvae were kept in the dark all the time (control, dark) or kept in the dark and illuminated for different durations (30 min, $2 \mathrm{~h}$ ). The same neurons were imaged at $24 \mathrm{~h}$ (top row) and at $72 \mathrm{~h}$ (bottom row) after illumination started. (B) Survival rates of $\mathrm{c} 1 \mathrm{da}$ neurons are reduced with $2 \mathrm{~h}$ illumination. About $10 \%$ of $\mathrm{c} 1 \mathrm{da}$ neuron imaged were found dead $72 \mathrm{~h}$ following $2 \mathrm{~h}$ caspase-LOV activation. (C-D) Quantifications of dendrite structures of c1da neurons following caspase-LOV activation, including total dendrite length (C) and total dendrite tip numbers (D). (E-F) Quantifications of changes in dendrite length (E) and tip numbers (F) of clda neurons over the $24 \mathrm{~h}-$ $72 \mathrm{~h}$ time period after caspase-LOV activation. (G-H) Quantifications for the percentage of eliminated (G) and added $(\mathrm{H})$ dendrite tips over the $24 \mathrm{~h}-72 \mathrm{~h}$ time period following caspase-LOV activation. Scale bars $=100 \mu \mathrm{m} . * \mathrm{p}<0.05, * * \mathrm{p}<0.01, * * * \mathrm{p}<0.001$, Kruskal-Wallis rank sum test with Dunn's post hoc test further adjusted by the Benjamini-Hochberg FDR method for multiple independent samples (B); one-way ANOVA with Tukey's post hoc test for multiple comparisons in $(\mathrm{C}-\mathrm{H})$. Error bars represent \pm SEM. $n=22-28$ neurons for each experimental condition and timepoint. 


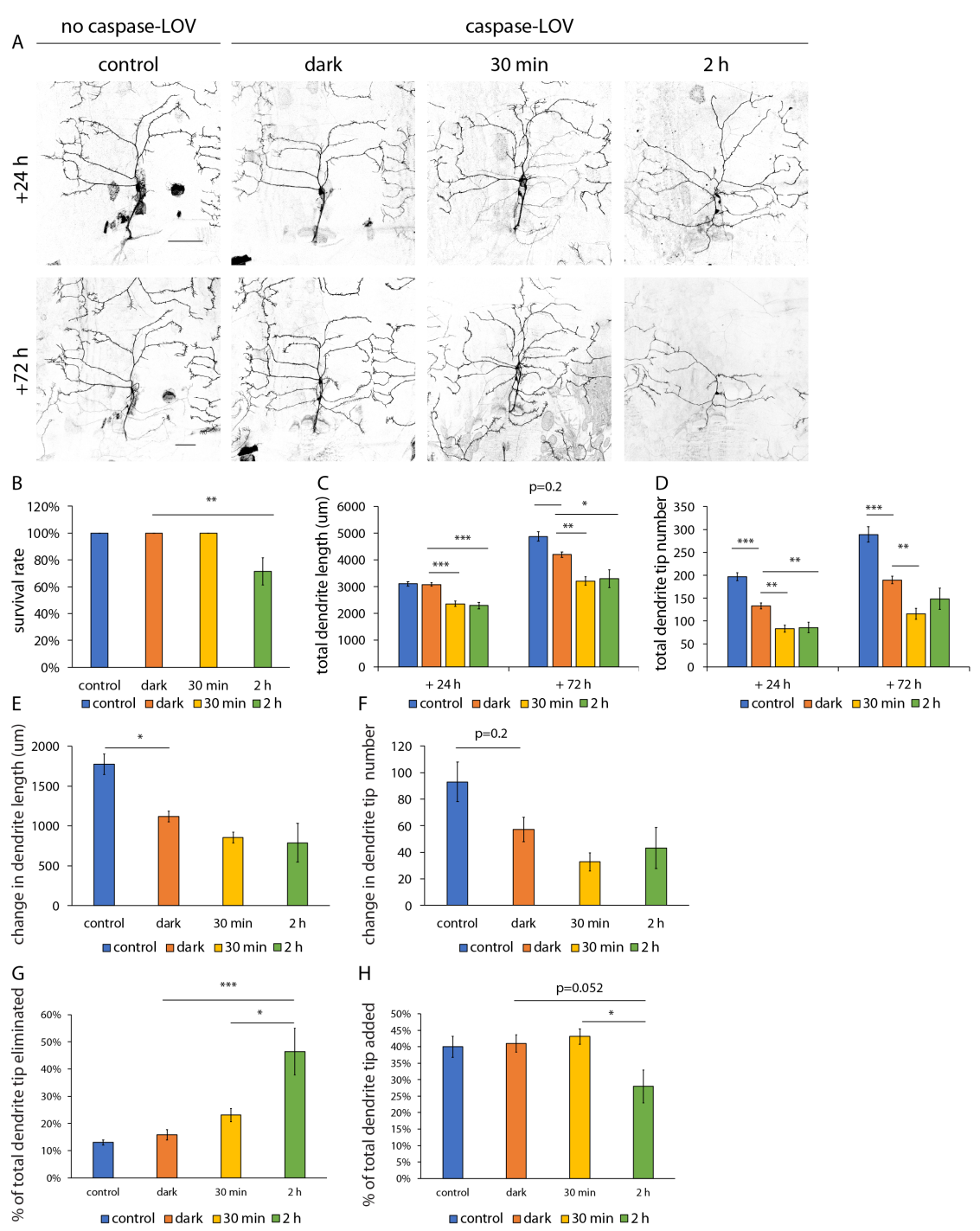

Figure 4. Class III ddaF neurons can sustain mild caspase-LOV activation and repair by adding new branches. (A) Representative images of c3da neurons expressing just UAS-tdTOM (control) or UAStdTOM and UAS-caspase-LOV (dark, $30 \mathrm{~min}, 2 \mathrm{~h}$ ) driven by ppk ${ }^{19-12}$-GAL4 along with Repo-Gal80. Larvae were kept in the dark all the time (control, dark) or kept in the dark and illuminated for different durations (30min, $2 \mathrm{~h}$ ). The same neurons were imaged at $24 \mathrm{~h}$ (top row) and at $72 \mathrm{~h}$ (bottom row) after illumination started. (B) Survival rates of c3da neurons are reduced with $2 \mathrm{~h}$ illumination. (C-D) Quantifications of dendrite structures of $\mathrm{c} 3 \mathrm{da}$ neurons following caspase-LOV activation, including total dendrite length (C) and total dendrite tip numbers (D). (E-F) Quantifications of change in dendrite length (E) and tip numbers (F) of c3da neurons over the $24 \mathrm{~h}-72 \mathrm{~h}$ time period after caspase-LOV activation. (G$\mathrm{H})$ Quantifications for the percentage of eliminated $(\mathrm{G})$ and added $(\mathrm{H})$ dendrite tips over the $24 \mathrm{~h}-72 \mathrm{~h}$ time period following caspase-LOV activation. Scale bars $=100 \mu \mathrm{m} .{ }^{*} \mathrm{p}<0.05,{ }^{*} \mathrm{p}<0.01,{ }^{*} * \mathrm{p}<0.001$, KruskalWallis rank sum test with Dunn's post hoc test further adjusted by the Benjamini-Hochberg FDR method for multiple independent samples (B); one-way ANOVA with Tukey's post hoc test for multiple 
A



C

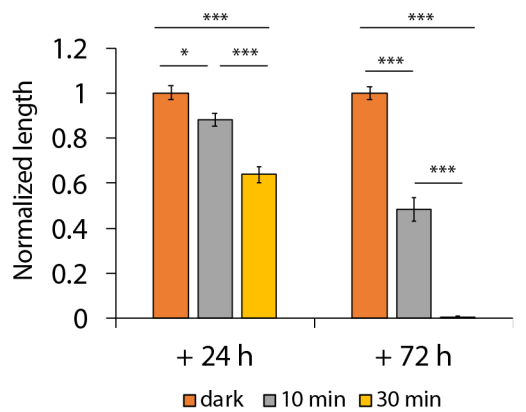

B

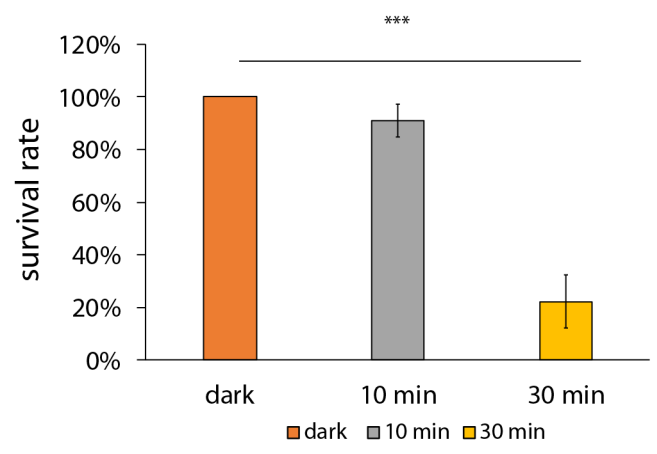

E





994

995

996

997

998

999

1000

1001

1002

1003

1004

1005

1006

1007

1008

1009

1010

1011

1012

1013

1014

1015

1016

1017

1018
Figure 5 - figure supplement 1. Degeneration and repair in the c4da neurons of tester animals. (A) Representative images of c4da neurons expressing UAS-luciferase and UAS-caspase-LOV driven by ppkgal4 and labeled with ppk-tdGFP. Larvae were illuminated for $10 \mathrm{~min}$ or $30 \mathrm{~min}$ and imaged following the protocol in Fig. 1A. (B) Survival rates of c4da neurons decreased significantly upon 30 min illumination. (C-E) Quantifications of dendrite structures, including normalized length (C), normalized tip numbers (D), and normalized percentage of territory covered (E) of c4da neurons kept in the dark, illuminated for $10 \mathrm{~min}$ or illuminated for $30 \mathrm{~min}$. The dendrite degeneration in the surviving c4da neurons is worse when illumination is extended. Scale bars $=100 \mu \mathrm{m} .{ }^{*} \mathrm{p}<0.05,{ }^{*} \mathrm{p}<0.01,{ }^{* * *} \mathrm{p}<0.001$, Kruskal-Wallis rank sum test with Dunn's post hoc test further adjusted by the Benjamini-Hochberg FDR method for multiple independent samples (B); one-way ANOVA with Tukey's post hoc test for multiple comparisons in (C-E). Error bars represent \pm SEM. $n=16-24$ neurons for each experimental condition and timepoint. 
A
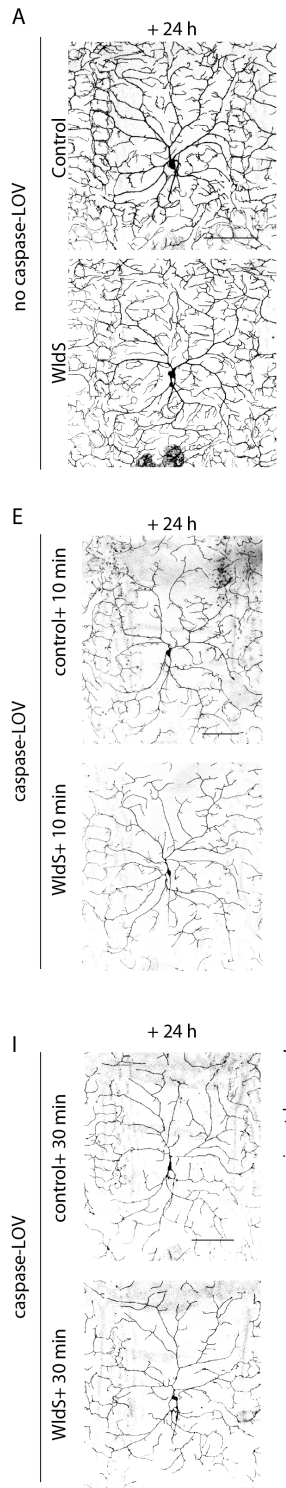
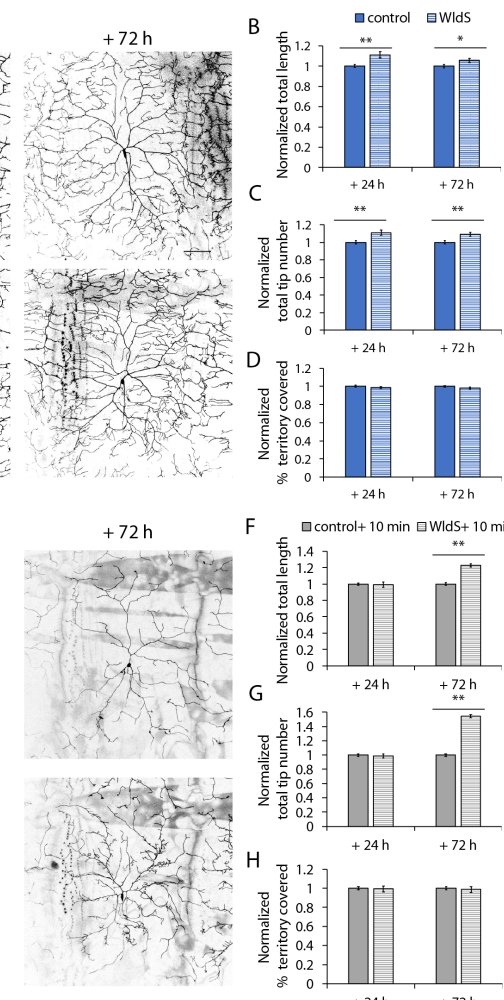

F घcontrol+10 min $\boxminus$ WIdS $+10 \mathrm{~min}$



$\mathrm{K}$
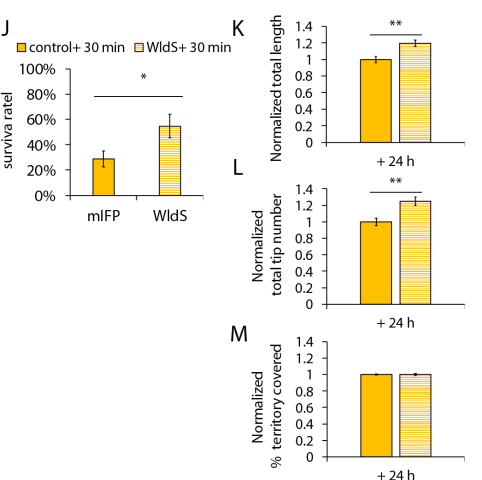

Figure 5. Wld ${ }^{\mathrm{S}}$ expressing neurons retain longer and more dendrites during development and upon caspase-3 induced neurodegeneration. (A) Representative images of c4da neurons labeled by ppk-tdGFP with ppk-Gal4 driving expression of UAS-mIFP-2A-HO1 (control) or UAS-Wld ${ }^{\mathrm{S}}$ (Wld ${ }^{\mathrm{S}}$ ). (B-D) Quantifications of dendrite structures, including normalized length (B), normalized tip numbers (C), and normalized percentage of territory covered (D) of c4da neurons. (E, I) Representative images of c4da neurons expressing ppk-tdGFP and caspase-LOV with UAS-mIFP-2A-HO1 (control) or UAS-Wld ${ }^{\mathrm{S}}$ driven by ppk-Gal4. Larva were kept in the dark and illuminated for $10 \mathrm{~min}$ (E) or $30 \mathrm{~min}$ (I) at $48 \mathrm{~h}$ after egg lay and imaged $24 \mathrm{~h}$ or $72 \mathrm{~h}$ afterward. (F-H) Quantifications of dendrite structures, including normalized length $(F)$, normalized tip numbers $(\mathrm{G})$, and normalized percentage of territory covered $(\mathrm{H})$ of c4da neurons illuminated for $10 \mathrm{~min}$. (J-M) Quantifications of survival rate $(\mathrm{J})$ and dendrite structures, including normalized length $(\mathrm{K})$, normalized tip numbers (L), and normalized percentage of territory covered (M) of c4da neurons illuminated for $30 \mathrm{~min}$. Scale bars $=100 \mu \mathrm{m}$. * $\mathrm{p}<0.05$, ** $\mathrm{p}<0.01, * * * \mathrm{p}<0.001$, Student's $t$ test in (B-D, F-H, K-M), Kruskal-Wallis rank sum test with Dunn's post hoc test further adjusted by the Benjamini-Hochberg FDR method for multiple independent samples (J); Error bars represent \pm SEM. $\mathrm{n} \geq 29$ neurons for each experimental condition and timepoint. 

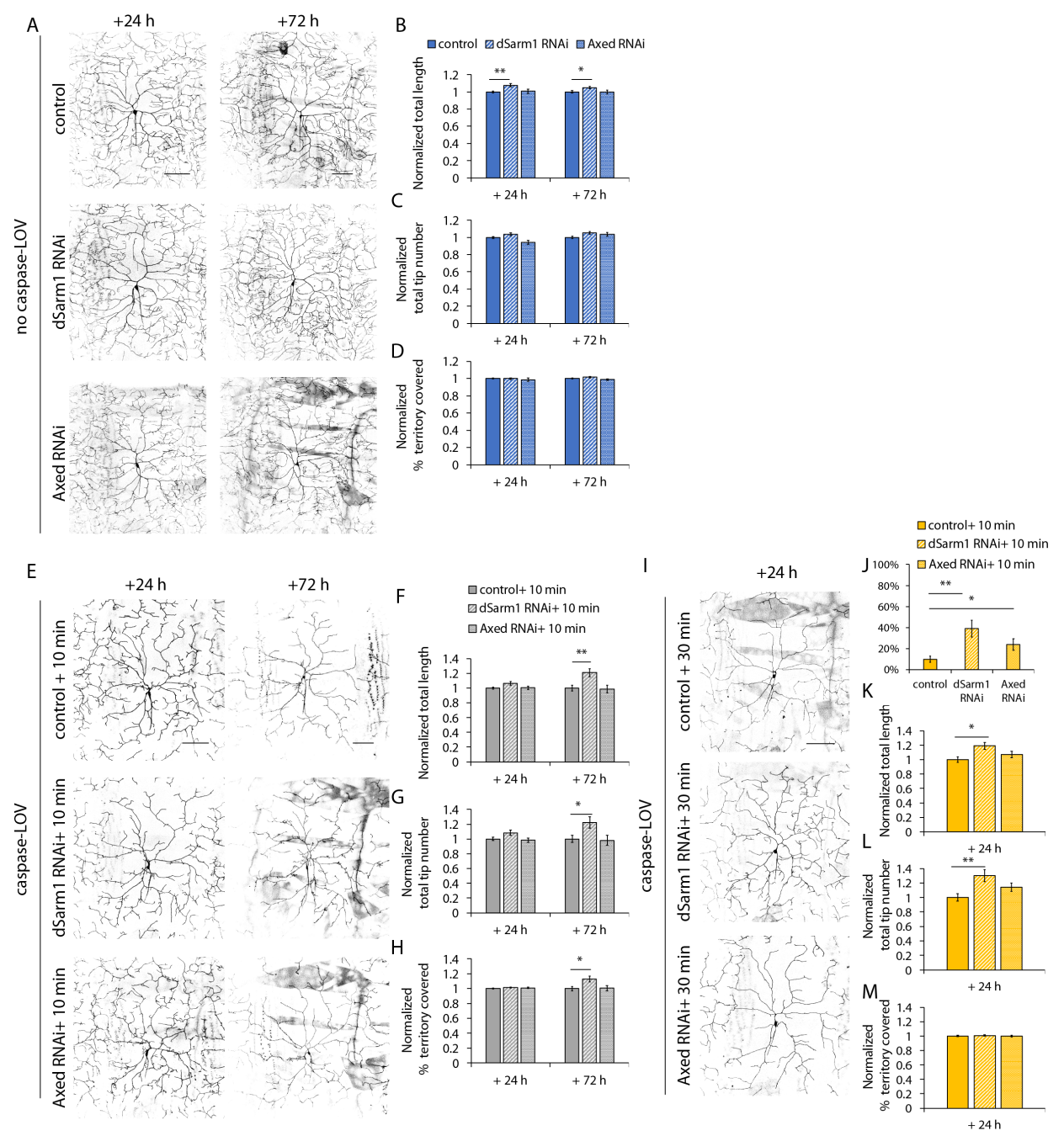

Figure 6. dSarm1 knockdown improves neuronal survival and allows neurons to retain longer dendrites throughout development and upon caspase-3 induced degeneration, while Axed knockdown only increases neuronal survival. (A) Representative images of c4da neurons labeled by ppktdGFP with ppk-Gal4 driving expression of UAS-luciferase (control), UAS-dSarm1 RNAi, or UAS-Axed RNAi. C4da neurons expressing dSarm1 RNAi have longer dendrites at early development $(+24 \mathrm{~h})$ and the dendrite length remains long until late development $(+72 \mathrm{~h}$ ). Knockdown of Axed in c4da neurons does not affect dendrite development. (B-D) Quantifications of dendrite structures, including normalized length (B), normalized tip numbers (C), and normalized percentage of territory covered (D) of c4da neurons. (E, I) Representative images of c4da neurons expressing ppk-tdGFP and UAS-caspase-LOV and UASluciferase (control), UAS-dSarm1 RNAi, or UAS-Axed RNAi driven by ppk-Gal4. Larva were kept in the dark and illuminated for $10 \mathrm{~min}$ (E) or $30 \mathrm{~min}$ (I) at $48 \mathrm{~h}$ after egg laying and imaged after $24 \mathrm{~h}$ or $72 \mathrm{~h}$. (F$\mathrm{H})$ Quantifications of dendrite structures, including normalized length $(\mathrm{F})$, normalized tip numbers $(\mathrm{G})$, and normalized percentage of territory covered $(\mathrm{H})$ of c4da neurons illuminated for $10 \mathrm{~min}$. (J-M) Quantifications of survival rate $(\mathrm{J})$ and dendrite structures, including normalized length $(\mathrm{K})$, normalized tip numbers (L), and normalized percentage of territory covered (M), of c4da neurons illuminated for $30 \mathrm{~min}$. Scale bars $=100 \mu \mathrm{m} . * \mathrm{p}<0.05, * * \mathrm{p}<0.01, * * * \mathrm{p}<0.001$, one way ANOVA with Tukey's post hoc test for multiple comparison in (B-D, F-H, K-M), Kruskal-Wallis rank sum test with Dunn's post hoc test further adjusted by the Benjamini-Hochberg FDR method for multiple independent samples (J); Error bars represent \pm SEM. $\mathrm{n} \geq 29$ neurons for each experimental condition and timepoint. 

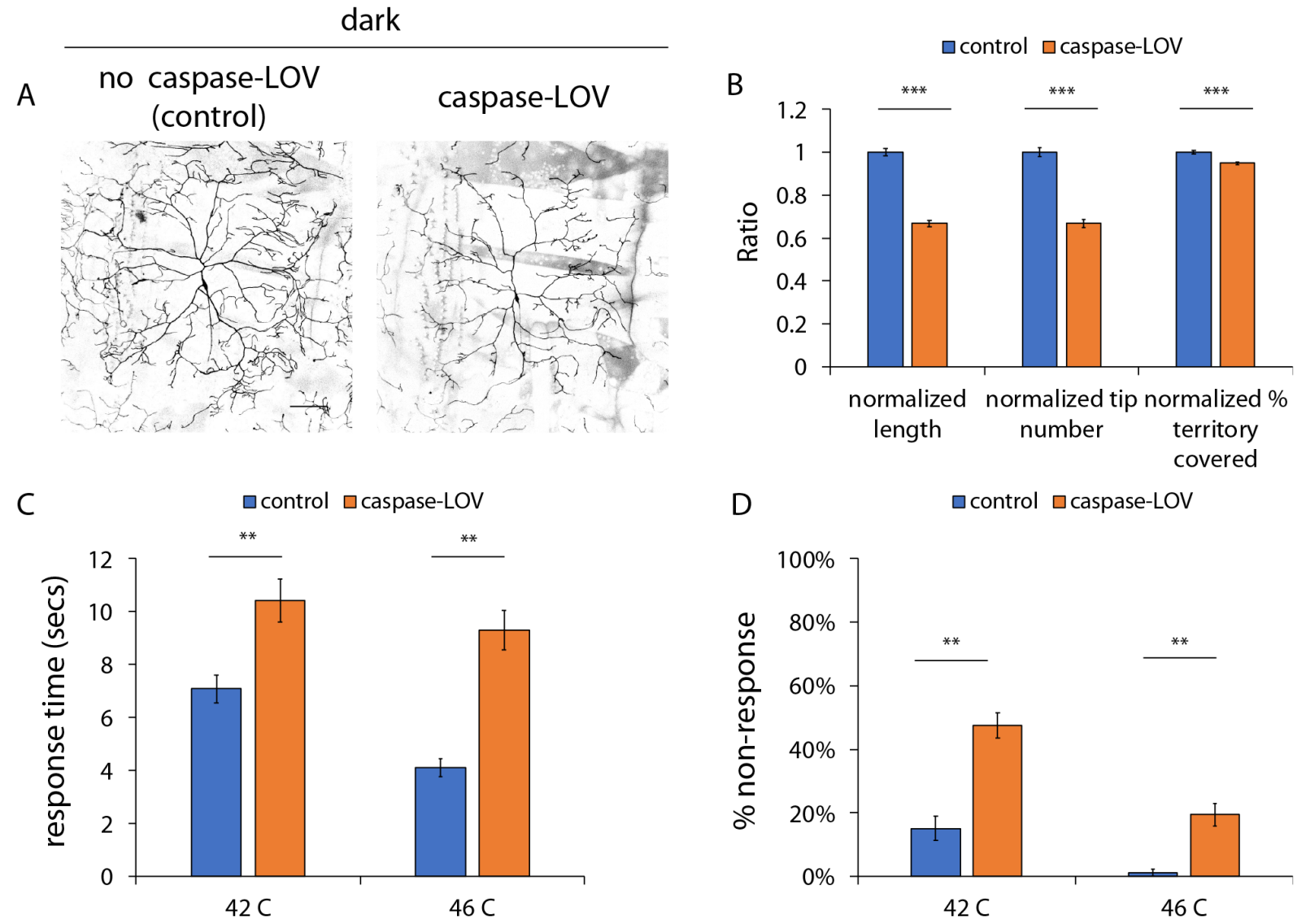

Figure 7. Activation of caspase-LOV in c4da neurons impaired the thermal nociceptive behavior. (A) Representative images of c4da neurons expressing UAS-tdTOM and UAS-luciferase (control) or UAStdTOM and UAS-caspase-LOV (caspase-LOV) driven by ppk-GAL4. Larvae are raised in the dark. C4da neurons expressing caspase-LOV have significant reductions in dendrite length, tip numbers and percentage of territory covered compared to control neurons at third-instar wandering stage. (B) Quantifications of dendrite structures, including normalized length (left), normalized tip numbers (middle), and normalized percentage of territory covered (right) of c4da neurons. (C-D) Aversive responses of third-instar wandering larvae in response to nocifensive temperature at $42^{\circ} \mathrm{C}$ and $46^{\circ} \mathrm{C}$ is affected by low-level caspase-LOV activation in the dark with longer response times (C) and a higher percentage of non-responders (D). Animals were classified as "non-responder" if the larva did not initiate the rolling behavior within $20 \mathrm{~s}$ of heated thermal probe touching the body wall. Scale bars $=100 \mu \mathrm{m}$. $* \mathrm{p}<0.05, * * \mathrm{p}<0.01, * * * \mathrm{p}<0.001$, Student's $t$ test in (B-D). Error bars represent \pm SEM. B: $\mathrm{n}=31$ (control) or 39 (caspase-LOV) neurons were tested. C-D: $n \geq 85$ animals were tested for each genotype and temperature. 
A
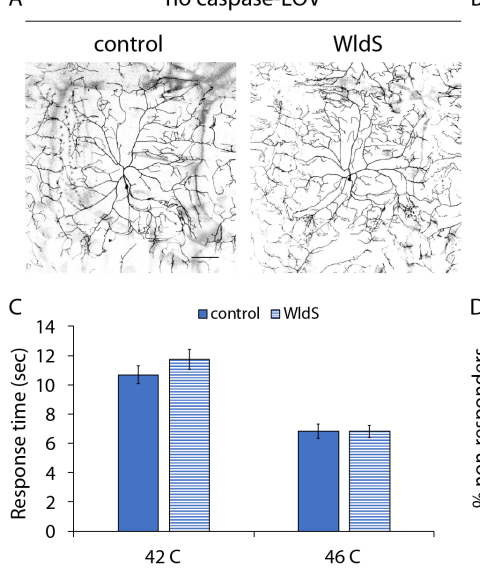

$\mathrm{E}$

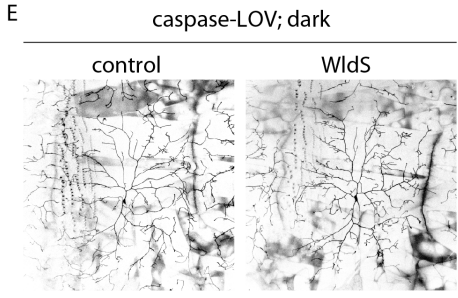

G



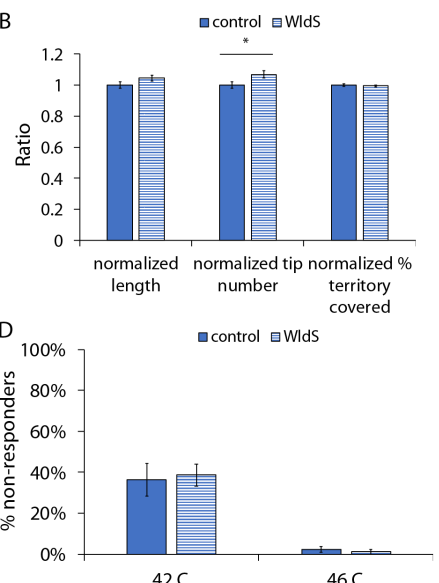

F

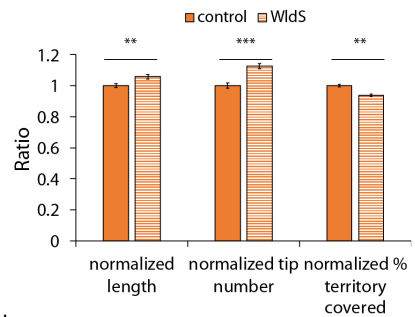

$\mathrm{H}$



Figure 8. Wld ${ }^{\mathrm{S}}$ can reduce caspase-3 induced dendrite degeneration and impairment in the thermal nocifensive behavior. (A) Representative images of $\mathrm{c} 4 \mathrm{da}$ neurons expressing tdTOM and UAS-mIFP-2AHO1 (control) or UAS-tdTOM and UAS-Wld ${ }^{\mathrm{S}}\left(\mathrm{Wld}^{\mathrm{S}}\right)$ driven by ppk-GAL4. Larvae are raised in the dark. C4da neurons expressing $\mathrm{Wld}^{\mathrm{S}}$ have significant increases in total dendrite tip numbers compared to control neurons during dendrite development at the third-instar wandering stage. (B) Quantifications of dendrite structures, including normalized length (left), normalized tip numbers (middle), and normalized percentage of territory covered (right) of c4da neurons. (C-D) $\mathrm{Wld}^{\mathrm{S}}$ expression on its own in c4da neurons does not change the response time $(\mathrm{C})$ nor the percentage of animals that do not respond to nocifensive temperatures of $42^{\circ} \mathrm{C}$ and $46^{\circ} \mathrm{C}$ (D). (E) Representative images of c4da neurons expressing tdTOM, and caspase-LOV and UAS-mIFP-2A-HO1 (control) or UAS-tdTOM, UAS-caspase-LOV, and UAS-Wld ${ }^{\mathrm{S}}$ driven by ppkGAL4. Larvae are raised in the dark. C4da neurons expressing $\mathrm{Wld}^{\mathrm{S}}$ can protect neurons from dendrite degeneration induced by low-level caspase-LOV activation in the dark as shown by significant increases in both dendrite length and tip numbers. There are reductions in the percentage of territory covered in Wld ${ }^{\mathrm{S}}$ expressing c4da neurons compared to control neurons. (F) Quantifications of dendrite structures, including normalized length (left), normalized tip numbers (middle), and normalized percentage of territory covered (right) of c4da neurons. (G-H) Slower thermal nocifensive response induced by caspase- 3 can be partially rescued by expression of $\mathrm{Wld}^{\mathrm{S}}$ in $\mathrm{c} 4 \mathrm{da}$ neurons. Wld $\mathrm{S}$ expression in animals with low-level caspase-LOV activation in the dark leads to a decreased response time $(\mathrm{G})$ at $46^{\circ} \mathrm{C}$ and the lower percentage of nonresponding animals $(\mathrm{H})$ in response to nocifensive temperature at $42^{\circ} \mathrm{C}$ and $46^{\circ} \mathrm{C}$. Scale bars $=100 \mu \mathrm{m}$. $*$ $\mathrm{p}<0.05, * * \mathrm{p}<0.01, * * * \mathrm{p}<0.001$, Student's $t$ test in (B-D, F-H). Error bars represent \pm SEM. B, F: $\mathrm{n} \geq 51$ neurons for each genotype. C-D, G-H: $\mathrm{n} \geq 75$ animals were tested for each genotype and temperature. 



Figure 9. Knockdown of dSarm1 or Axed reduces the thermal nocifensive behavior. (A) Representative images of c4da neurons expressing UAS-tdTOM and UAS-luciferase (control), UAStdTOM and UAS-dSarm1 RNAi (dSarm1 RNAi), or UAS-tdTOM and UAS-Axed RNAi (Axed RNAi) driven by ppk-GAL4. Knockdown of dSarm1 or Axed by RNAi in c4da neurons does not change dendrite structures compared to control neurons during dendrite development at the third-instar wandering stage. All larvae are raised in the dark. (B) Quantifications of dendrite structures, including normalized length (left), normalized tip numbers (middle), and normalized percentage of territory covered (right) of c4da neurons. (C-D) Third-instar wandering larvae expressing dSarm1 RNAi in c4da neurons responded slower (C) and had a higher percentage of non-responding animals at $42^{\circ} \mathrm{C}(\mathrm{D})$. Knockdown of Axed also increases the percentage of animals with no response to $42^{\circ} \mathrm{C}(\mathrm{D})$. (E) Knockdown of dSarml or Axed by RNAi in c4da neurons does not change dendrite degeneration induced by caspase-LOV activation in the dark compared to control neurons at the third-instar wandering stage. (F) Quantifications of dendrite structures, including normalized length (left), normalized tip numbers (middle), and normalized percentage of territory covered (right) of c4da neurons. (G-H) Slower thermal nocifensive response induced by caspase-3 is worsen when dSarm1 and Axed are knocked down in c4da neurons. When dSarm1 expression is knocked down in these neurons, there is an increased response time at $42^{\circ} \mathrm{C}(\mathrm{G})$. When either dSarm 1 or Axed are knocked down, there is a higher percentage of non-responding animals when probed at $42^{\circ} \mathrm{C}$ and at $46^{\circ} \mathrm{C}(\mathrm{H})$. Scale bars $=100 \mu \mathrm{m} . * \mathrm{p}<0.05, * * \mathrm{p}<0.01, * * * \mathrm{p}<0.001$, one-way ANOVA with Tukey's post hoc test for multiple comparison in (B-D, F-H). Error bars represent \pm SEM. B, F: $\mathrm{n} \geq 44$ neurons for each genotype. C-D, G-H: $\mathrm{n} \geq 72$ animals were tested for each genotype and temperature. 